\title{
GRADIENT BOUNDS AND RIGIDITY RESULTS FOR SINGULAR, DEGENERATE, ANISOTROPIC PARTIAL DIFFERENTIAL EQUATIONS
}

\author{
MATTEO COZZI ${ }^{(1,2)}, \operatorname{ALBERTO~FARINA}^{(1,3)}, \operatorname{AND~ENRICO~VALDINOCI~}^{(2,4,5)}$
}

(1) - Laboratoire Amiénois de Mathématique Fondamentale et Appliquée UMR CNRS 7352, Université Picardie "Jules Verne" 33 Rue St Leu, 80039 Amiens (France).

(2) - Dipartimento di Matematica "Federigo Enriques" Università degli studi di Milano, Via Saldini 50, I-20133 Milano (Italy).

(3) - Institut "Camille Jordan"

UMR CNRS 5208, Université "Claude Bernard" Lyon I 43 Boulevard du 11 novembre 1918, 69622 Villeurbanne cedex (France).

(4) - Istituto di Matematica Applicata e Tecnologie Informatiche "Enrico Magenes" Consiglio Nazionale delle Ricerche Via Ferrata 1, I-27100 Pavia (Italy).

(5) - Weierstraß Institut für Angewandte Analysis und Stochastik Mohrenstraße 39, D-10117 Berlin (Germany).

E-mail addresses: matteo.cozzi@unimi.it, alberto.farina@u-picardie.fr, enrico@math.utexas.edu

ABSTRACT: We consider the Wulff-type energy functional

$$
\mathscr{W}_{\Omega}(u):=\int_{\Omega} B(H(\nabla u(x)))-F(u(x)) d x,
$$

where $B$ is positive, monotone and convex, and $H$ is positive homogeneous of degree 1 . The critical points of this functional satisfy a possibly singular or degenerate, quasilinear equation in an anisotropic medium.

We prove that the gradient of the solution is bounded at any point by the potential $F(u)$ and we deduce several rigidity and symmetry properties.

\section{INTRODUCTION AND MAIN RESULTS}

We consider here a variational problem in an anisotropic medium. The physical motivation we have in mind comes from some well-established models of surface energy, see for instance [T78, G06] and references therein for a classical introduction to the topic.

Surface energy arises since the microscopic environment of the interface of a medium is different from the one in the bulk of the substance. In many concrete cases, such as for the common cooking salt, the different behavior depends significantly on the space direction and so these anisotropic surface energies have now become very popular in metallurgy and crystallography, see e.g. W01, D44, AC77. Applications to crystal growth and thermodynamics are discussed in [MBK77, C84, TCH92] and in [G93, respectively.

Other applications of related anisotropic models occur in noise-removal procedures in digital image processing, crystalline mean curvature flows and crystalline fracture theory, see e.g. NP99, BNP01a, BNP01b, EO04, OBGXY05, and references therein. See also [FM91, C04 for anisotropic problems related to the Willmore functional and CS09, WX11] for elliptic anisotropic systems inspired by fluidodynamics. We defer the interested reader to Appendix $\mathbb{C}$ for some deeper physical insights. 
Of course, besides this surface energy, the medium may also be subject to exterior forces and the total energy functional is in this case the sum of an anisotropic surface energy plus a potential term. More precisely, the mathematical framework we work in is inspired by the Wulff crystal construction (see pages 571-573 in [T78]) and it may be formally introduced as follows.

Given a domain $\Omega \subseteq \mathbb{R}^{n}$, with $n \geqslant 2$, consider the functional

$$
\mathscr{W}_{\Omega}(u):=\int_{\Omega} B(H(\nabla u(x)))-F(u(x)) d x .
$$

Here, $B$ denotes a function of class $C_{\text {loc }}^{3, \beta}((0,+\infty)) \cap C^{1}([0,+\infty))$, with $\beta \in(0,1)$, such that $B(0)=B^{\prime}(0)=0$ and

$$
B(t), B^{\prime}(t), B^{\prime \prime}(t)>0 \text { for any } t \in(0,+\infty) .
$$

Also, $H: \mathbb{R}^{n} \rightarrow \mathbb{R}$ is a positive homogeneous function of degree 1 , of class $C_{\text {loc }}^{3, \beta}\left(\mathbb{R}^{n} \backslash\{0\}\right)$, with

$$
H(\xi)>0 \text { for any } \xi \in \mathbb{R}^{n} \backslash\{0\} .
$$

Notice that, being $H$ homogeneous and defined at the origin, it necessarily holds $H(0)=0$. Finally, take $F \in C_{\text {loc }}^{2, \beta}(\mathbb{R})$ and assume that either $(\mathrm{A})$ or $(\mathrm{B})$ is satisfied, where:

(A) There exist $p>1, \kappa \in[0,1)$ and positive $\gamma, \Gamma$ such that, for any $\xi \in \mathbb{R}^{n} \backslash\{0\}, \zeta \in \mathbb{R}^{n}$,

$$
[\operatorname{Hess}(B \circ H)(\xi)]_{i j} \zeta_{i} \zeta_{j} \geqslant \gamma(\kappa+|\xi|)^{p-2}|\zeta|^{2},
$$

and

$$
\sum_{i, j=1}^{n}\left|[\operatorname{Hess}(B \circ H)(\xi)]_{i j}\right| \leqslant \Gamma(\kappa+|\xi|)^{p-2} .
$$

(B) The composition $B \circ H$ is of class $C_{\text {loc }}^{3, \beta}\left(\mathbb{R}^{n}\right)$ and for any $K>0$ there exist a positive constant $\gamma$ such that, for any $\xi, \zeta \in \mathbb{R}^{n}$, with $|\xi| \leqslant K$, we have

$$
[\operatorname{Hess}(B \circ H)(\xi)]_{i j} \zeta_{i} \zeta_{j} \geqslant \gamma|\zeta|^{2} \text {. }
$$

Here above and throughout the paper, the summation convention for repeated subscripts is used, unless differently specified. Critical points of $\mathscr{W}_{\Omega}$ weakly satisfy the Euler-Lagrange equation

$$
\frac{\partial}{\partial x_{i}}\left(B^{\prime}(H(\nabla u)) H_{i}(\nabla u)\right)+F^{\prime}(u)=0,
$$

where $H_{i}(\xi)=\partial_{\xi_{i}} H(\xi)$.

The model we consider is indeed very general and it allows at the same time an anisotropic dependence on the space variable and a possible singularity or degeneracy of the diffusion operator. For instance, we can take into account the following examples of $B$ :

$$
B(t)=\frac{\left(\kappa^{2}+t^{2}\right)^{p / 2}-\kappa^{p}}{p} \quad \text { and } \quad B(t)=\sqrt{1+t^{2}}-1
$$

with $p>1$, and $\kappa \geqslant 0$.

Such choices are related to the anisotropic p-Laplace equation

$$
\operatorname{div}\left(H^{p-1}(\nabla u) \nabla H(\nabla u)\right)+F^{\prime}(u)=0,
$$

obtained by taking $B(t)=t^{p} / p$, and the anisotropic minimal surface equation

$$
\operatorname{div}\left(\frac{H(\nabla u) \nabla H(\nabla u)}{\sqrt{1+H^{2}(\nabla u)}}\right)+F^{\prime}(u)=0 .
$$

In particular, when $H(\xi)=|\xi|$, equations (1.6) and (1.7) reduce respectively to the classical $p$-Laplace and minimal surface equations. 
As an example of anisotropic $H$ one may consider the function

$$
H(\xi)=\sqrt{\langle M \xi, \xi\rangle}
$$

with $M \in \operatorname{Mat}_{n}(\mathbb{R})$ symmetric and positive definite. We stress that the combination of such a $H$ along with $B$ as in (1.5), with $\kappa>0$ in the $p$-Laplacian case, actually produces an operator that satisfies hypothesis (B). In Appendix B we prove that indeed this is the only possible choice for $H$, in the framework of assumption (B).

We refer instead to Appendix $\mathrm{A}$ for the construction of a rather general anisotropic function $H$ which is not necessarily a norm.

Given $u: \mathbb{R}^{n} \rightarrow \mathbb{R}$, we define

$$
c_{u}:=\sup \left\{F(r): r \in\left[\inf _{\mathbb{R}^{n}} u, \sup _{\mathbb{R}^{n}} u\right]\right\} .
$$

The quantity $c_{u}$ is an important potential gauge. Indeed, the nonlinearity $f$ defines the potential $F$ uniquely up to an additive constant. An appropriate choice of this constant makes the results that we are going to present as sharp as possible: roughly speaking, this gauge consists in taking $F(u)-c_{u}$ as effective potential (notice that such potential is nonpositive on the solution $u$ ). Furthermore we are able to identify explicitly the value of the quantity $c_{u}$, as showed in Theorem 1.6 .

Our main results are a pointwise estimate on the gradient of the solution, from which we deduce some rigidity and symmetry properties (in particular, we obtain one-dimensional Euclidean symmetry and Liouville-type results).

Thus, the first result we present is a pointwise bound on the gradient in terms of the effective potential. Notice that classical elliptic estimates provide bounds of the gradient in either Hölder or Lebesgue norms, but do not give any pointwise information in general. In dimension 1, the pointwise estimate that we present reduces to the classical Energy Conservation Law.

In higher dimension, estimates of this kind were given first by [M85] for the semilinear equation

$$
\Delta u+F^{\prime}(u)=0
$$

with $F \leqslant 0$ (this case is comprised in our setting by choosing $H(\xi)=|\xi|, B(t)=t^{2} / 2$ ). Then, CGS94 extended such estimates to the quasilinear case

$$
\operatorname{div}\left(\Phi^{\prime}\left(|\nabla u|^{2}\right) \nabla u\right)+F^{\prime}(u)=0
$$

with $F \leqslant 0$ (this is a particular case in our framework given by $H(\xi)=|\xi|, B(t)=$ $\left.(1 / 2) \Phi\left(t^{2}\right)\right)$.

Recently, some attention has been given to the case of anisotropic media and the first pointwise estimate in this setting was given in [FV13] for equations of the type

$$
\operatorname{div}(H(\nabla u) \nabla H(\nabla u))+F^{\prime}(u)=0
$$

(again, this is a particular case for us by taking $B(t)=t^{2} / 2$ ).

Our purpose is to extend the previous results to the general case of anisotropic media with possible nonlinearities, singularities and nondegeneracies in the diffusion operator (indeed, the function $H$ encodes the anisotropy of the medium and the function $B$ the possible degeneracies of the operator). The precise statement of our pointwise bound is the following:

Theorem 1.1. Assume that one of the following conditions is valid:

(i) Assumption (A) holds and $u \in L^{\infty}\left(\mathbb{R}^{n}\right) \cap W_{\text {loc }}^{1, p}\left(\mathbb{R}^{n}\right)$ is a weak solution of (1.4) in $\mathbb{R}^{n}$;

(ii) Assumption (B) holds and $u \in W^{1, \infty}\left(\mathbb{R}^{n}\right)$ weakly solves (1.4) in $\mathbb{R}^{n}$.

Then, for any $x \in \mathbb{R}^{n}$,

$$
B^{\prime}(H(\nabla u(x))) H(\nabla u(x))-B(H(\nabla u(x))) \leqslant c_{u}-F(u(x)) .
$$

Moreover, if there exists $x_{0} \in \mathbb{R}^{n}$ such that

$$
\nabla u\left(x_{0}\right) \neq 0
$$


and

$$
B^{\prime}\left(H\left(\nabla u\left(x_{0}\right)\right)\right) H\left(\nabla u\left(x_{0}\right)\right)-B\left(H\left(\nabla u\left(x_{0}\right)\right)\right)=c_{u}-F\left(u\left(x_{0}\right)\right),
$$

then

$$
B^{\prime}(H(\nabla u)) H(\nabla u)-B(H(\nabla u))=c_{u}-F(u) .
$$

on the whole connected component of $\{\nabla u \neq 0\}$ containing $x_{0}$.

Now we state our main symmetry result, according to which the equality in (1.10) implies that the solution only depends on one Euclidean variable (in particular, the classical and anisotropic curvatures of the level sets vanish identically):

Theorem 1.2. Let $u$ be as in Theorem 1.1. Suppose that there exists $x_{0} \in \mathbb{R}^{n}$ such that $\nabla u\left(x_{0}\right) \neq 0$ and (1.10) holds true.

Then there exist $u_{0}: \mathbb{R} \rightarrow \mathbb{R}$ and $\omega \in S^{n-1}$ such that $u(x)=u_{0}(\omega \cdot x)$ for any $x$ in the connected component $\mathscr{S}$ of $\{\nabla u \neq 0\}$ containing $x_{0}$, and the level sets of $u$ in $\mathscr{S}$ are affine hyperplanes.

We observe that one-dimensional solutions $u(x)=u_{0}(\omega \cdot x)$ of (1.4) satisfy the ordinary differential equation

$$
B^{\prime \prime}\left(H\left(\omega \dot{u}_{0}\right)\right) H^{2}(\omega) \ddot{u}_{0}+F^{\prime}\left(u_{0}\right)=0 .
$$

Equivalently, (1.11) reduces in this case to the Energy Conservation Law

$$
b\left(H\left(\omega \dot{u}_{0}\right)\right)=c_{u_{0}}-F\left(u_{0}\right),
$$

where $b(t):=B^{\prime}(t) t-B(t)$.

Theorem 1.2 was proved in the isotropic setting in [CGS94 under the additional assumption that $F \leqslant 0$, and in the planar, anisotropic setting in [FV13. Therefore Theorem 1.2 is new in the anisotropic setting even for cases that are not singular or degenerate (e.g. for $\left.B(t)=t^{2} / 2\right)$. We stress in particular that the proof of this result is different from the ones in CGS94, FV13] since we exploit for the first time the consequences of the vanishing of the $P$-function by taking into account explicitly an appropriate remainder term: indeed, such $P$-function is not only a subsolution of a suitable equation, but it is also a solution of an equation with a term that has a sign and that vanishes when $P$ is constant (see the forthcoming equation (4.4) for details).

Under some further (but natural) assumptions, Theorem 1.2 holds globally in the whole of the space, as next results point out:

Theorem 1.3. Let $u$ be as in Theorem 1.1 with condition (ii) in force. Assume that there exists $x_{0} \in \mathbb{R}^{n}$ such that $\nabla u\left(x_{0}\right) \neq 0$ and (1.10) holds true.

Then there exist $u_{0}: \mathbb{R} \rightarrow \mathbb{R}$ and $\omega \in S^{n-1}$ such that $u(x)=u_{0}(\omega \cdot x)$ for any $x \in \mathbb{R}^{n}$.

We observe that the assumptions of Theorem 1.3 are satisfied by many cases of interest, such as the minimal surface and the regularized $p$-Laplace equations (e.g. with $B$ as in (1.5) with $\kappa>0$ ). A global version of Theorem 1.3 which encompasses all the cases under consideration is given by the following result:

Theorem 1.4. Let $u$ be as in Theorem 1.1 and assume that (1.11) holds in the whole of $\mathbb{R}^{n}$.

Then there exist $u_{0}: \mathbb{R} \rightarrow \mathbb{R}$ and $\omega \in S^{n-1}$ such that $u(x)=u_{0}(\omega \cdot x)$ for any $x \in \mathbb{R}^{n}$.

Differently from CGS94 in which results similar to Theorems 1.3 and 1.4 were obtained in the isotropic setting with a different method, we do not need to assume any sign assumption on $F$. The next is a Liouville-type result that shows that the solution is constant if the effective potential and its derivative vanish at some point (the isotropic case was dealt with in CGS94, CFV12]). 
Theorem 1.5. Let $u$ be as in Theorem 1.1. If condition (i) of Theorem 1.1 is in force, with $\kappa=0$ and $p>2$, assume in addition that, given a value $r \in \mathbb{R}$ such that $F(r)=c_{u}$ and $F^{\prime}(r)=0$, we have

$$
\left|F^{\prime}(\sigma)\right|=O\left(|\sigma-r|^{p-1}\right) \text { as } \sigma \rightarrow r .
$$

If there exists a point $x_{0} \in \mathbb{R}^{n}$ for which $F\left(u\left(x_{0}\right)\right)=c_{u}$ and $F^{\prime}\left(u\left(x_{0}\right)\right)=0$, then $u$ is constant.

Notice that condition (1.13) cannot be removed from Theorem 1.5, since, without such assumption, one can construct smooth, non-constant, one-dimensional solutions: see Proposition 7.2 in [FSV08] for an explicit, non-constant example in which (1.13) is not satisfied and

$$
F\left(\min _{\mathbb{R}^{n}} u\right)=F\left(\max _{\mathbb{R}^{n}} u\right)=c_{u} \text { and } F^{\prime}\left(\min _{\mathbb{R}^{n}} u\right)=F^{\prime}\left(\max _{\mathbb{R}^{n}} u\right)=0 .
$$

We also remark that, in principle, to obtain $c_{u}$ one is expected to know all the values of the solution $u$ and to compute the potential out of them. Next result shows in fact that this is not necessary, and that $c_{u}$ may be computed once we know only the infimum and the supremum of the solution (the isotropic case was dealt with in [FV10]):

Theorem 1.6. Let $u$ and $F$ be as in Theorem 1.5. Then,

$$
c_{u}=\max \left\{F\left(\inf _{\mathbb{R}^{n}} u\right), F\left(\sup _{\mathbb{R}^{n}} u\right)\right\} .
$$

Furthermore, if there exists $y_{0} \in \mathbb{R}^{n}$ such that $F\left(u\left(y_{0}\right)\right)=c_{u}$, then

$$
\text { either } u\left(y_{0}\right)=\inf _{\mathbb{R}^{n}} u \text { or } u\left(y_{0}\right)=\sup _{\mathbb{R}^{n}} u \text {. }
$$

The paper is organized as follows. First, in Section 2 we collect some technical and ancillary results. The regularity of the solutions is briefly tackled in Section 3. The proof of Theorem 1.1 relies on a $P$-function argument that is discussed in Section 4 (roughly speaking, one has to check that a suitable energy function is a subsolution of a partial differential equation and to use the Maximum Principle to obtain the desired bound). The proofs of the main results are collected in Sections 5 10. In Appendices $\mathrm{A}$ and B, respectively, we present an example of function $H$ which is not a norm and the proof of the fact that any $H$ fulfilling assumption (B) is of the form (1.8). Finally, some physical interpretations of the so-called Wulff shape of $H$ are briefly discussed in Appendix C.

\section{Some PRELIMINARY RESUlts}

The first part of this section is mainly devoted to some elementary facts about positive homogeneous functions. We mostly provide only the statements, referring to [FV13] for the omitted proofs.

We recall that a function $H: \mathbb{R}^{n} \backslash\{0\} \rightarrow \mathbb{R}$ is said to be positive homogeneous of degree $d \in \mathbb{R}$ if $H(t \xi)=t^{d} H(\xi)$, for any $t>0$ and $\xi \in \mathbb{R}^{n} \backslash\{0\}$.

Lemma 2.1. If $H \in C^{m}\left(\mathbb{R}^{n} \backslash\{0\}\right)$ is positive homogeneous of degree $d$ and $\alpha \in \mathbb{N}^{n}$ with $\alpha_{1}+\cdots+\alpha_{n}=m$, then $\partial^{\alpha} H$ is positive homogeneous of degree $d-m$.

Notice that the corresponding result proved in FV13, Lemma 2, only deals with integer degrees. Nevertheless, the proof works the same way considering a real degree $d$.

Next, we establish the identities commonly used in the course of the main proofs.

Lemma 2.2. If $H \in C^{3}\left(\mathbb{R}^{n} \backslash\{0\}\right)$ is positive homogeneous of degree 1 , we have that

$$
\begin{aligned}
H_{i}(\xi) \xi_{i} & =H(\xi), \\
H_{i j}(\xi) \xi_{i} & =0, \\
H_{i j k}(\xi) \xi_{i} & =-H_{j k}(\xi) .
\end{aligned}
$$


Now, we justify the smoothness of $H$ needed to write (1.4) and to use the regularity theory:

Lemma 2.3. Let $H \in C^{1}\left(\mathbb{R}^{n} \backslash\{0\}\right)$ be a positive homogeneous function of degree d admitting non-negative values and $B \in C^{1}([0,+\infty))$, with $B(0)=0$. Assume that either $d>1$ or $d=1$ and $B^{\prime}(0)=0$. Then $H$ can be extended by setting $H(0):=0$ to a continuous function, such that $B \circ H \in C^{1}\left(\mathbb{R}^{n}\right)$ and

$$
\partial_{i}(B \circ H)(0)=0=\lim _{x \rightarrow 0} B^{\prime}(H(x)) H_{i}(x) .
$$

Proof. Setting $H(0):=0$ clearly transforms $H$ into a continuous function on the whole of $\mathbb{R}^{n}$, since $|H(\xi)| \leqslant|\xi|^{d} \sup _{\mathrm{S}^{n-1}}|H|$, for any $\xi \in \mathbb{R}^{n} \backslash\{0\}$. Moreover, $B \circ H \in C^{1}\left(\mathbb{R}^{n} \backslash\{0\}\right)$, and

$$
\begin{aligned}
\partial_{i}(B \circ H)(0) & =\lim _{t \rightarrow 0} \frac{B\left(H\left(t e_{i}\right)\right)}{t}=\lim _{t \rightarrow 0^{ \pm}} \frac{B\left(H\left( \pm|t| e_{i}\right)\right)}{t} \\
& =\lim _{t \rightarrow 0^{ \pm}} \frac{B\left(|t|^{d} H\left( \pm e_{i}\right)\right)}{t}= \pm H\left( \pm e_{i}\right)^{\frac{1}{d}} \lim _{s \rightarrow 0^{+}} \frac{B(s)}{s} s^{\frac{d-1}{d}} \\
& = \pm H\left( \pm e_{i}\right)^{\frac{1}{d}} B^{\prime}(0) \lim _{s \rightarrow 0^{+}} s^{\frac{d-1}{d}}=0 .
\end{aligned}
$$

On the other hand, by Lemma 2.1, $H_{i}(x)=|x|^{d-1} H_{i}(x /|x|)$ for any $x \in \mathbb{R}^{n} \backslash\{0\}$, and so

$$
\begin{aligned}
\lim _{x \rightarrow 0}\left|B^{\prime}(H(x)) H_{i}(x)\right| & \leqslant \sup _{S^{n-1}}\left|H_{i}\right| \lim _{x \rightarrow 0}|x|^{d-1}\left|B^{\prime}\left(|x|^{d} H\left(\frac{x}{|x|}\right)\right)\right| \\
& =\left|B^{\prime}(0)\right| \sup _{S^{n-1}}\left|H_{i}\right| \lim _{x \rightarrow 0}|x|^{d-1}=0,
\end{aligned}
$$

as desired.

Then, we have the following characterization of the positive definiteness of the composition $B \circ H$.

Lemma 2.4. Let $B \in C^{2}((0,+\infty))$ be a function satisfying (1.2) and $H \in C^{2}\left(\mathbb{R}^{n} \backslash\{0\}\right)$ be positive homogeneous of degree 1 satisfying (1.3). Then, the following two statements are equivalent:

(i) Hess $(B \circ H)$ is positive definite in $\mathbb{R}^{n} \backslash\{0\}$;

(ii) The restriction of Hess $(H)(\xi)$ to $\xi^{\perp}$ is a positive definite endomorphism $\xi^{\perp} \rightarrow \xi^{\perp}$, for all $\xi \in S^{n-1}$.

Proof. Our argument is an adaptation of the proof of Proposition 2 on page 102 of WX11. The case covered there is the one with $B(t)=t^{2}$.

First, we prove that $(i)$ implies $(i i)$. Fix $\xi \in S^{n-1}$. Assumption $(i)$ is equivalent to

$$
\left[B^{\prime \prime}(H(\xi)) H_{i}(\xi) H_{j}(\xi)+B^{\prime}(H(\xi)) H_{i j}(\xi)\right] \zeta_{i} \zeta_{j}>0 \text { for any } \zeta \in \mathbb{R}^{n} \backslash\{0\} .
$$

Observe now that $\nabla H(\xi)$ cannot be orthogonal to $\xi$, since, by (2.1),$H_{i}(\xi) \xi_{i}=H(\xi)>0$. Therefore, $\nabla H(\xi)^{\perp}$ and $\xi$ span the whole of $\mathbb{R}^{n}$. Letting now $V \in \xi^{\perp}$, we write

$$
V=\zeta+\lambda \xi, \quad \text { for some } \zeta \in \nabla H(\xi)^{\perp} \backslash\{0\}, \lambda \in \mathbb{R} .
$$

Applying (2.4) with $\zeta=V-\lambda \xi$ and using (2.2), we get

$$
\begin{aligned}
0 & <\left[B^{\prime \prime}(H(\xi)) H_{i}(\xi) H_{j}(\xi)+B^{\prime}(H(\xi)) H_{i j}(\xi)\right] \zeta_{i} \zeta_{j}=B^{\prime}(H(\xi)) H_{i j}(\xi) \zeta_{i} \zeta_{j} \\
& =B^{\prime}(H(\xi)) H_{i j}(\xi)\left(V_{i}-\lambda \xi_{i}\right)\left(V_{j}-\lambda \xi_{j}\right)=B^{\prime}(H(\xi)) H_{i j}(\xi) V_{i} V_{j},
\end{aligned}
$$

which, by (1.2), gives (ii).

Conversely, assume that (ii) holds. Let $V \in \mathbb{R}^{n} \backslash\{0\}$ and decompose it into $V=\eta+\lambda \xi$, for $\eta \in \xi^{\perp}, \lambda \in \mathbb{R}$. By (2.2), (1.2) and (ii) we obtain

$$
\begin{aligned}
& {\left[B^{\prime \prime}(H(\xi)) H_{i}(\xi) H_{j}(\xi)+B^{\prime}(H(\xi)) H_{i j}(\xi)\right] V_{i} V_{j}} \\
& \quad=B^{\prime \prime}(H(\xi)) H_{i}(\xi) H_{j}(\xi) V_{i} V_{j}+B^{\prime}(H(\xi)) H_{i j}(\xi)\left(\eta_{i}+\lambda \xi_{i}\right)\left(\eta_{j}+\lambda \xi_{j}\right) \\
& \quad=B^{\prime \prime}(H(\xi))[V \cdot \nabla H(\xi)]^{2}+B^{\prime}(H(\xi)) H_{i j}(\xi) \eta_{i} \eta_{j} \geqslant B^{\prime}(H(\xi)) H_{i j}(\xi) \eta_{i} \eta_{j}>0
\end{aligned}
$$


if $\eta \neq 0$. If on the other hand $\eta=0$, i.e. $V=\lambda \xi$ with $\lambda \neq 0$, then, using (2.1) and (2.2),

$$
\begin{aligned}
& {\left[B^{\prime \prime}(H(\xi)) H_{i}(\xi) H_{j}(\xi)+B^{\prime}(H(\xi)) H_{i j}(\xi)\right] V_{i} V_{j}} \\
& \quad=\lambda^{2}\left[B^{\prime \prime}(H(\xi)) H_{i}(\xi) H_{j}(\xi) \xi_{i} \xi_{j}+B^{\prime}(H(\xi)) H_{i j}(\xi) \xi_{i} \xi_{j}\right]=\lambda^{2} B^{\prime \prime}(H(\xi)) H^{2}(\xi)>0,
\end{aligned}
$$

so that $(i)$ is proved.

Next, we have a result ensuring the convexity of $H$. We point out that this actually comes as a corollary of Lemma 2.4 and Lemma 2.2 together.

Lemma 2.5. Let $H \in C^{2}\left(\mathbb{R}^{n} \backslash\{0\}\right)$ be a positive homogeneous function of degree 1 satisfying (1.3) and $B \in C^{2}((0,+\infty))$ be such (1.2) holds. Assume also Hess $(B \circ H)$ to be positive definite in $\mathbb{R}^{n} \backslash\{0\}$. Then $H$ is convex and

$$
H_{i j}(\xi) \eta_{i} \eta_{j} \geqslant 0 \quad \text { for any } \xi \in \mathbb{R}^{n} \backslash\{0\} \text { and } \eta \in \mathbb{R}^{n} .
$$

Following is a linear algebra result that is crucial for the subsequent proofs of Proposition 4.1 and Theorem 1.2 .

Proposition 2.6. Let $H$ and $B$ as in the statement of Lemma 2.5. Then, given any matrix $\left\{c_{i j}\right\}_{i, j \in\{1, \ldots, n\}}$, we have

$$
H_{i j}(\xi) H_{k \ell}(\xi) c_{i k} c_{j \ell} \geqslant 0 \quad \text { for any } \xi \in \mathbb{R}^{n} \backslash\{0\} .
$$

Moreover, assume that equality holds in (2.6) for a vector $\xi=\left(\xi_{1}, \ldots, \xi_{n}\right) \in \mathbb{R}^{n} \backslash\{0\}$ such that

$$
\xi_{1}=\cdots=\xi_{n-1}=0
$$

Then 1

$$
c_{i^{\prime} j^{\prime}}=0 \text { for any } i^{\prime}, j^{\prime} \in\{1, \ldots, n-1\} .
$$

Proof. We follow the argument given at the end of the proof of Proposition 1 of [FV13]. By points (ii) in Lemma 2.4 and (2.2), we know that

$$
\begin{aligned}
& \operatorname{Hess}(H)(\xi) \text { has } n-1 \text { strictly positive eigenvalues and one null eigenvalue } \\
& \text { (the latter corresponding to the eigenvector } \xi \text { ). }
\end{aligned}
$$

Therefore, we can diagonalize it via an orthogonal matrix $\left\{M_{i j}\right\}_{i, j \in\{1, \ldots, n\}}$, by writing

$$
H_{i j}=M_{p i} \lambda_{p} M_{p j}, \text { with } \lambda_{1} \geqslant \ldots \geqslant \lambda_{n-1}>\lambda_{n}=0 .
$$

So, setting

$$
\vartheta_{p r}:=M_{p i} M_{r m} c_{i m}
$$

for fixed $p$ and $r$, we have that

$$
0 \leqslant\left(\vartheta_{p r}\right)^{2}=\left(M_{p i} M_{r k} c_{i k}\right)\left(M_{p j} M_{r \ell} c_{j \ell}\right)=M_{p i} M_{p j} M_{r k} M_{r \ell} c_{i k} c_{j \ell} .
$$

Now, multiply by $\lambda_{p} \lambda_{r}$ and sum over $p$ and $r$. We get

$$
0 \leqslant \lambda_{p} \lambda_{r}\left(\vartheta_{p r}\right)^{2}=M_{p i} \lambda_{p} M_{p j} M_{r k} \lambda_{r} M_{r \ell} c_{i k} c_{j \ell}=H_{i j} H_{k \ell} c_{i k} c_{j \ell},
$$

which proves (2.6).

Now we assume (2.7) and we suppose that equality holds in (2.6). We claim that

$$
M_{n i^{\prime}}=0 \text { for any } i^{\prime} \in\{1, \ldots, n-1\} .
$$

For this, we use a classical linear algebra procedure: we define $w_{i}:=M_{n i}$ and we consider the vector $w=\left(w_{1}, \ldots, w_{n}\right)$. We exploit (2.10) and we have, for any $j \in\{1, \ldots, n\}$,

$$
\begin{array}{r}
(\operatorname{Hess}(H)(\xi) w)_{j}=H_{j k} w_{k}=M_{i j} \lambda_{i} M_{i k} w_{k}=M_{i j} \lambda_{i} M_{i k} M_{n k} \\
=M_{i j} \lambda_{i} \delta_{i n}=M_{n j} \lambda_{n}=0=(0 w)_{j} .
\end{array}
$$

\footnotetext{
${ }^{1}$ To avoid confusion, we use indices like $i$ ranging in $\{1, \ldots, n\}$ and like $i^{\prime}$ ranging in $\{1, \ldots, n-1\}$.
} 
That is, $w$ is an eigenvector for $\operatorname{Hess}(H)(\xi)$ and so, by (2.9),$w$ is parallel to $\xi$. Thus, by (2.7), $w$ is parallel to $(0, \ldots, 0,1)$ and so $w_{i^{\prime}}=0$ for any $i^{\prime} \in\{1, \ldots, n-1\}$, proving (2.13).

Now, if equality holds in (2.6), then (2.12) gives that

$$
0=\lambda_{p} \lambda_{r}\left(\vartheta_{p r}\right)^{2} .
$$

Consequently, by (2.10), we obtain that

$$
\vartheta_{p^{\prime} r^{\prime}}=0 \text { for any } p^{\prime}, r^{\prime} \in\{1, \ldots, n-1\} .
$$

Hence, we invert (2.11) and we obtain that

$$
M_{p j} M_{r k} \vartheta_{p r}=M_{p j} M_{p i} M_{r k} M_{r m} c_{i m}=\delta_{i j} \delta_{m k} c_{i m}=c_{j k}
$$

for any $j, k \in\{1, \ldots, n\}$. So, recalling (2.13) and (2.14), we have, for any $j^{\prime}, k^{\prime} \in\{1, \ldots, n-$ $1\}$,

$$
c_{j^{\prime} k^{\prime}}=M_{p j^{\prime}} M_{r k^{\prime}} \vartheta_{p r}=M_{p^{\prime} j^{\prime}} M_{r^{\prime} k^{\prime}} \vartheta_{p^{\prime} r^{\prime}}=0,
$$

where the indices $p^{\prime}, r^{\prime}$ are summed over $\{1, \ldots, n-1\}$.

Now we collect two technical inequalities concerning function $B$ which will be used in the proofs of Theorems 1.4 and 1.5 .

Lemma 2.7. Let $B \in C^{2}((0,+\infty)) \cap C^{0}([0,+\infty))$ be a function satisfying $B(0)=0$ and (1.2). Then,

$$
B^{\prime}(t) t-B(t)>0
$$

for any $t>0$.

Proof. For any $t>0$ set

$$
b(t):=B^{\prime}(t) t-B(t) .
$$

Clearly, $b \in C^{1}((0,+\infty))$. By differentiation we get, for $t>0$,

$$
b^{\prime}(t)=B^{\prime \prime}(t) t+B^{\prime}(t)-B^{\prime}(t)=B^{\prime \prime}(t) t>0,
$$

since $B^{\prime \prime}(t)$ is positive. Thus, $b$ is strictly increasing and so

$$
b(t)>b\left(0^{+}\right)=0, \quad \text { for any } t>0,
$$

which proves the lemma.

Lemma 2.8. Let $B \in C^{2}((0,+\infty)) \cap C^{1}([0,+\infty))$ be such $B(0)=0$ and $H \in C^{2}\left(\mathbb{R}^{n} \backslash\{0\}\right)$ be a positive homogeneous function of degree 1 satisfying (1.3). Assume that they either satisfy $(A)$ or $(B)$. Then, for any $M>0$, there exists $\varepsilon>0$ such that

$$
B^{\prime}(t) t-B(t) \geqslant \varepsilon t^{p^{*}} \text { for any } t \in[0, M],
$$

where

$$
p^{*}=\left\{\begin{array}{l}
p \quad \text { if }(A) \text { holds with } \kappa=0 \\
2 \quad \text { otherwise. }
\end{array}\right.
$$

Proof. Let $M>0, b$ be as in (2.16) and $\varepsilon>0$ to be determined later. Define, for any non-negative $t$,

$$
E(t):=b(t)-\varepsilon t^{p^{*}}=B^{\prime}(t) t-B(t)-\varepsilon t^{p^{*}} .
$$

If we prove that

$$
E^{\prime}(t) \geqslant 0 \text { for any } t \in(0, M],
$$

is true, then we are done, since in this case we have

$$
E(t) \geqslant E(0)=0 \text { for any } t \in(0, M],
$$


which leads directly to (2.17). To show that (2.18) holds, fix $t \in(0, M]$ and choose $\xi \in$ $\mathbb{R}^{n} \backslash\{0\}$ in a way that $t=H(\xi)$. Notice that this can surely be done by taking $\xi:=t H^{-1}(\nu) \nu$, for any $\nu \in \mathbb{R}^{n} \backslash\{0\}$. In particular, we have that

$$
|\xi|=H^{-1}\left(\frac{\xi}{|\xi|}\right) t \leqslant h t \leqslant h M,
$$

if we set $h^{-1}=\inf _{S^{n-1}} H>0$. Applying now the first formula of (A) with $\zeta=\xi$ we get

$$
\begin{aligned}
c_{1}(\kappa+|\xi|)^{p-2}|\xi|^{2} & \leqslant[\operatorname{Hess}(B \circ H)(\xi)]_{i j} \xi_{i} \xi_{j}=\partial_{\xi_{j}}\left(B^{\prime}(H(\xi)) H_{i}(\xi)\right) \xi_{i} \xi_{j} \\
& =\left(B^{\prime \prime}(H(\xi)) H_{i}(\xi) H_{j}(\xi)+B^{\prime}(H(\xi)) H_{i j}(\xi)\right) \xi_{i} \xi_{j} \\
& =B^{\prime \prime}(H(\xi)) H^{2}(\xi),
\end{aligned}
$$

where in the last equality we used (2.1) and (2.2). By the homogeneity of $H$ we thus may conclude that

$$
B^{\prime \prime}(H(\xi)) \geqslant c_{1}(\kappa+|\xi|)^{p-2}|\xi|^{2} H^{-2}(\xi) \geqslant c(\kappa+|\xi|)^{p-2},
$$

for some positive constant $c$. Now, if $\kappa>0$ and $p \geqslant 2$, we drop $|\xi|$ in the last parenthesis, getting

$$
B^{\prime \prime}(H(\xi)) \geqslant c \kappa^{p-2} .
$$

If $\kappa>0$ but $1<p<2$, then by (2.19) we have

$$
B^{\prime \prime}(H(\xi)) \geqslant c(\kappa+h M)^{p-2} .
$$

If on the other hand $\kappa=0$, we simply rewrite (2.20), obtaining

$$
B^{\prime \prime}(H(\xi)) \geqslant c|\xi|^{p-2} \geqslant c^{\prime} H^{p-2}(\xi),
$$

for some positive constant $c^{\prime}$. Collecting these three cases and making explicit the dependence on $t$, we get

$$
B^{\prime \prime}(t) \geqslant c^{\prime \prime} t^{p^{*}-2},
$$

for some positive constant $c^{\prime \prime}$. An analogous computation shows that the same result holds also when (B) is in force. By (2.21) and choosing $\varepsilon$ small enough, we compute

$$
E^{\prime}(t)=B^{\prime \prime}(t) t-\varepsilon p^{*} t^{p^{*}-1} \geqslant\left(c^{\prime \prime}-\varepsilon p^{*}\right) t^{p^{*}-1} \geqslant 0
$$

which gives (2.18).

Notice that, in the setting of the paper, Lemma 2.7 actually comes as a corollary of Lemma 2.8. Nevertheless, we preferred to state them independently one to the other, since the hypotheses required by the first do not involve the function $H$ at all.

Finally, we present a lemma ensuring the continuity of the second derivative of $B$ at the origin starting from some regularity assumptions on the composition $B \circ H$. The framework in which this result is meant to be set is that of hypothesis (B) and, in fact, explicit use of it will be made in Section 7 .

Lemma 2.9. Let $H \in C^{2}\left(\mathbb{R}^{n} \backslash\{0\}\right)$ be a positive homogeneous function of degree 1 satisfying (1.3) and $B \in C^{1}([0,+\infty)) \cap C^{2}((0,+\infty))$, with $B(0)=B^{\prime}(0)=0$. Assume in addition that $B \circ H$ has some pure second derivative, say, the first, continuous at the origin. Then, $B \in C^{2}([0,+\infty))$ with

$$
B^{\prime \prime}(0)=H^{-2}\left(e_{1}\right) \frac{\partial^{2}(B \circ H)}{\partial \xi_{1}^{2}}(0) .
$$

In particular, this holds if $B \circ H \in C^{2}\left(\mathbb{R}^{n}\right)$. 
Proof. Since, for every $\xi \neq 0$,

$$
\frac{\partial^{2}(B \circ H)}{\partial \xi_{1}^{2}}(\xi)=B^{\prime \prime}(H(\xi)) H_{1}^{2}(\xi)+B^{\prime}(H(\xi)) H_{11}(\xi),
$$

by choosing $\xi=t e_{1}$, with $t>0$, and the homogeneity properties of $H$ we obtain

$$
\frac{\partial^{2}(B \circ H)}{\partial \xi_{1}^{2}}\left(t e_{1}\right)=B^{\prime \prime}\left(t H\left(e_{1}\right)\right) H_{1}^{2}\left(e_{1}\right)+\frac{B^{\prime}\left(t H\left(e_{1}\right)\right)}{t} H_{11}\left(e_{1}\right) .
$$

Now, observe that

$$
H_{1}\left(e_{1}\right)=\nabla H\left(e_{1}\right) \cdot e_{1}=H\left(e_{1}\right)>0
$$

by (2.1) and

$$
H_{11}\left(e_{1}\right)=\nabla H_{1}\left(e_{1}\right) \cdot e_{1}=0,
$$

by (2.2). Therefore, by (2.23) we get

$$
B^{\prime \prime}\left(t H\left(e_{1}\right)\right)=H^{-2}\left(e_{1}\right) \frac{\partial^{2}(B \circ H)}{\partial \xi_{1}^{2}}\left(t e_{1}\right),
$$

which yields (2.22) by passing to the limit as $t \rightarrow 0^{+}$.

\section{Regularity of the SOlutions}

In this short section we point out some regularity properties of the weak solutions of (1.4).

Proposition 3.1. Let $u$ be as in Theorem 1.1. Then, given any $x_{0} \in \mathbb{R}^{n}$ and $R \in(0,1)$, there exist $\alpha \in(0,1)$ and $C>0$, depending only on $n, R,\|u\|_{L^{\infty}\left(\mathbb{R}^{n}\right)}$ and the constants involved in $(A)$ or $(B)$, so that

$$
\begin{gathered}
\|\nabla u\|_{L^{\infty}\left(\mathbb{R}^{n}\right)} \leqslant C, \\
|\nabla u(x)-\nabla u(y)| \leqslant C R^{-\alpha}|x-y|^{\alpha},
\end{gathered}
$$

for any $x, y \in B_{R}\left(x_{0}\right)$. In particular, $u \in C_{\operatorname{loc}}^{1, \alpha}\left(\mathbb{R}^{n}\right)$, for such $\alpha$.

Proof. In case $(i)$ of Theorem 1.1 holds, we can apply Theorem 1 on page 127 of [T84. Notice that the ellipticity and growth conditions there required are satisfied by assumption (A) and the structure of equation (1.4). Condition (1.7) of T84 is also valid, due to the fact that $f$ is continuous and $u$ bounded. Finally, the locally boundedness of the gradient in [T84 could be easily extended to the whole of $\mathbb{R}^{n}$, giving (3.1). See also [DiB83].

If on the other hand ( $i i)$ is in force, then (3.1) is already satisfied. In order to obtain (3.2), the uniform ellipticity of the Hessian of $B(H(\nabla u))$ allows us to appeal to Theorem 1.1 on page 339 of [LU68] (notice that we know in addition that $u \in W_{\text {loc }}^{2,2}\left(\mathbb{R}^{n}\right)$ in this case, thanks to Proposition 1 in [T84, the boundedness of $\nabla u$ and the structural conditions in (ii)).

If we stay far from the points on which $\nabla u$ vanishes, then we can obtain even more regularity for $u$, as displayed by the following result:

Proposition 3.2. Let $u$ be as in Theorem 1.1. Then, for any $x \in \mathbb{R}^{n}$ with $\nabla u(x) \neq 0$ there exists $R>0$ and $\alpha \in(0,1)$ such that $u \in C^{3, \alpha}\left(B_{R}(x)\right)$.

In particular, we have that $u \in C^{3}(\{\nabla u \neq 0\})$.

Moreover, if assumption (ii) in Theorem 1.1 holds, we have the stronger conclusion that $u \in C_{\text {loc }}^{3, \alpha}\left(\mathbb{R}^{n}\right)$.

Proof. If $u$ satisfies $(i)$ of Theorem 1.1 and $x$ is as in the statement, then we may apply Theorem 6.4 on page 284 of [LU68] in some neighborhood of $x$ contained in $\{\nabla u \neq 0\}$, which exists due to the continuity of $\nabla u$ granted by Proposition 3.1. to obtain the thesis.

The same result also holds if condition $(i i)$ is valid, relying instead on Theorem 6.3 on page 283 of [LU68]. Note that, in this case, the non-degeneracy of $\nabla u$ is no longer required, obtaining that $u$ is actually of class $C_{\text {loc }}^{3, \alpha}$ on the whole of $\mathbb{R}^{n}$. 


\section{P-Function computations}

Now we perform a $P$-function argument, by showing that a suitable energy functional is a subsolution of a partial differential equation (in fact, it is a solution, with a remainder term which has a sign). Classical computations of this kind are in [P76, S81].

For the sake of briefness, in the following we will often adopt the notation $H=H(\nabla u)$, $H_{i}=\left(\partial_{i} H\right)(\nabla u), B=B(H(\nabla u)), B^{\prime}=B^{\prime}(H(\nabla u))$, etc.

Proposition 4.1. Let $u$ be as in Theorem 1.1. Set

$$
\begin{gathered}
G(r):=c_{u}-F(r) \text { for any } r \in \mathbb{R}, \\
a_{i j}:=B^{\prime \prime} H_{i} H_{j}+B^{\prime} H_{i j}, \quad d_{i j}:=a_{i j} / H,
\end{gathered}
$$

and

$$
P(u ; x):=B^{\prime}(H(\nabla u(x))) H(\nabla u(x))-B(H(\nabla u(x)))-G(u(x)) .
$$

Then,

$$
\left(d_{i j} P_{i}\right)_{j}-b_{k} P_{k}=\mathscr{R} \geqslant 0 \text { on }\{\nabla u \neq 0\},
$$

where

$$
b_{k}:=\frac{B^{\prime \prime \prime}}{B^{\prime \prime}} H^{-2} H_{\ell} P_{\ell} H_{k}+\left[\frac{B^{\prime \prime \prime}}{B^{\prime \prime}}+\frac{B^{\prime \prime}}{B^{\prime}}\right] G^{\prime} H^{-1} H_{k}+\left[\frac{B^{\prime} B^{\prime \prime \prime}}{\left(B^{\prime \prime}\right)^{2}}+1\right] H^{-2} H_{k \ell} P_{\ell}
$$

and $\mathscr{R}:=B^{\prime} B^{\prime \prime} H_{i j} H_{k \ell} u_{i k} u_{j \ell}$.

Proof. First of all, we point out that, by Proposition 3.2, $u$ is $C^{3}(\{\nabla u \neq 0\})$. We will therefore implicitly assume every calculation to be performed on $\{\nabla u \neq 0\}$. The computation is quite long and somehow delicate, but we provide full details of the argument for the facility of the reader. By differentiating (4.3), we get for any $i \in\{1, \ldots, n\}$

$$
P_{i}=B^{\prime \prime} H H_{k} u_{k i}+B^{\prime} H_{k} u_{k i}-B^{\prime} H_{k} u_{k i}-G^{\prime} u_{i}=B^{\prime \prime} H H_{k} u_{k i}-G^{\prime} u_{i} .
$$

Thus, recalling (4.2),

$$
\begin{aligned}
\left(d_{i j} P_{i}\right)_{j} & =\left(B^{\prime \prime} H H_{k} d_{i j} u_{k i}\right)_{j}-\left(G^{\prime} d_{i j} u_{i}\right)_{j} \\
& =\left(B^{\prime \prime} H_{k}\right)_{j} a_{i j} u_{k i}+B^{\prime \prime} H_{k}\left(a_{i j} u_{k i}\right)_{j}-\left(G^{\prime} d_{i j} u_{i}\right)_{j} .
\end{aligned}
$$

Next, observe that from (1.4) we have

$$
a_{i j} u_{i j}=G^{\prime}
$$

Being $u$ of class $C^{3}$, we compute for any $k$

$$
\begin{aligned}
& \left(a_{i j} u_{k i}\right)_{j}-\left(a_{i j} u_{i j}\right)_{k}=\left(a_{i j}\right)_{j} u_{k i}-\left(a_{i j}\right)_{k} u_{i j} \\
& \quad=\left[B^{\prime \prime \prime} H_{i} H_{j} H_{\ell}+B^{\prime \prime} H_{i \ell} H_{j}+B^{\prime \prime} H_{i} H_{j \ell}+B^{\prime \prime} H_{i j} H_{\ell}+B^{\prime} H_{i j \ell}\right] u_{j \ell} u_{k i} \\
& -\left[B^{\prime \prime \prime} H_{i} H_{j} H_{\ell}+B^{\prime \prime} H_{i \ell} H_{j}+B^{\prime \prime} H_{i} H_{j \ell}+B^{\prime \prime} H_{i j} H_{\ell}+B^{\prime} H_{i j \ell}\right] u_{k \ell} u_{i j}=0
\end{aligned}
$$

by interchanging the indices $i$ and $\ell$ in the last term. Therefore, using (4.8) we obtain

$$
\left(a_{i j} u_{k i}\right)_{j}=\left(a_{i j} u_{i j}\right)_{k}=\left(G^{\prime}\right)_{k}=G^{\prime \prime} u_{k} .
$$

Plugging this into (4.7) we have

$$
\begin{aligned}
\left(d_{i j} P_{i}\right)_{j} & =\left(B^{\prime \prime} H_{k}\right)_{j} a_{i j} u_{k i}+B^{\prime \prime} H_{k} G^{\prime \prime} u_{k}-\left(G^{\prime} d_{i j} u_{i}\right)_{j} \\
& =\left(B^{\prime \prime} H_{k}\right)_{j} a_{i j} u_{k i}+B^{\prime \prime} H_{k} G^{\prime \prime} u_{k}-G^{\prime \prime} d_{i j} u_{i} u_{j}-G^{\prime}\left(d_{i j} u_{i}\right)_{j} .
\end{aligned}
$$

Now, we collect the two terms containing $G^{\prime \prime}$, getting, by (2.1) and (2.2),

$$
\begin{aligned}
B^{\prime \prime} H_{k} G^{\prime \prime} u_{k}-G^{\prime \prime} d_{i j} u_{i} u_{j} & =G^{\prime \prime} H^{-1}\left[B^{\prime \prime} H H_{k} u_{k}-a_{i j} u_{i} u_{j}\right] \\
& =G^{\prime \prime} H^{-1}\left[B^{\prime \prime} H^{2}-B^{\prime \prime} H_{i} H_{j} u_{i} u_{j}-B^{\prime} H_{i j} u_{i} u_{j}\right] \\
& =G^{\prime \prime} H^{-1}\left[B^{\prime \prime} H^{2}-B^{\prime \prime} H^{2}-0\right]=0 .
\end{aligned}
$$


Hence, (4.10) becomes

$$
\begin{aligned}
\left(d_{i j} P_{i}\right)_{j} & =\left(B^{\prime \prime} H_{k}\right)_{j} a_{i j} u_{k i}-G^{\prime}\left(d_{i j} u_{i}\right)_{j} \\
& =\left(B^{\prime \prime} H_{k}\right)_{j} a_{i j} u_{k i}-G^{\prime}\left(d_{i j}\right)_{j} u_{i}-G^{\prime} d_{i j} u_{i j} \\
& =\left(B^{\prime \prime} H_{k}\right)_{j} a_{i j} u_{k i}-G^{\prime}\left(d_{i j}\right)_{j} u_{i}-\left(G^{\prime}\right)^{2} H^{-1}
\end{aligned}
$$

where in the last line we made use of (4.8). Appealing to (2.1), (2.2) and (2.3), we compute

$$
\begin{aligned}
\left(d_{i j}\right)_{j} u_{i}= & {\left[B^{\prime \prime} H^{-1} H_{i} H_{j}+B^{\prime} H^{-1} H_{i j}\right]_{j} u_{i} } \\
= & {\left[B^{\prime \prime \prime} H^{-1} H_{i} H_{j} H_{\ell}-B^{\prime \prime} H^{-2} H_{i} H_{j} H_{\ell}+B^{\prime \prime} H^{-1} H_{i \ell} H_{j}+B^{\prime \prime} H^{-1} H_{i} H_{j \ell}\right.} \\
& \left.+B^{\prime \prime} H^{-1} H_{i j} H_{\ell}-B^{\prime} H^{-2} H_{i j} H_{\ell}+B^{\prime} H^{-1} H_{i j \ell}\right] u_{j \ell} u_{i} \\
= & {\left[B^{\prime \prime \prime} H_{j} H_{\ell}-B^{\prime \prime} H^{-1} H_{j} H_{\ell}+0+B^{\prime \prime} H_{j \ell}+0-0-B^{\prime} H^{-1} H_{j \ell}\right] u_{j \ell} } \\
= & {\left[B^{\prime \prime \prime}-B^{\prime \prime} H^{-1}\right] H_{j} H_{\ell} u_{j \ell}+\left[B^{\prime \prime}-B^{\prime} H^{-1}\right] H_{j \ell} u_{j \ell} . }
\end{aligned}
$$

Writing explicitly (4.8)

$$
G^{\prime}=a_{i j} u_{i j}=B^{\prime \prime} H_{i} H_{j} u_{i j}+B^{\prime} H_{i j} u_{i j}
$$

we deduce

$$
H_{i j} u_{i j}=\left(B^{\prime}\right)^{-1}\left[G^{\prime}-B^{\prime \prime} H_{i} H_{j} u_{i j}\right] .
$$

By this equation, (4.12) becomes

$$
\begin{aligned}
\left(d_{i j}\right)_{j} u_{i} & =\left[B^{\prime \prime \prime}-B^{\prime \prime} H^{-1}\right] H_{j} H_{\ell} u_{j \ell}+\left(B^{\prime}\right)^{-1}\left[B^{\prime \prime}-B^{\prime} H^{-1}\right]\left[G^{\prime}-B^{\prime \prime} H_{j} H_{\ell} u_{j \ell}\right] \\
& =\left[B^{\prime \prime \prime}-\left(B^{\prime}\right)^{-1}\left(B^{\prime \prime}\right)^{2}\right] H_{j} H_{\ell} u_{j \ell}+G^{\prime}\left(B^{\prime}\right)^{-1}\left[B^{\prime \prime}-B^{\prime} H^{-1}\right] .
\end{aligned}
$$

Now, inverting (4.6), we get

$$
H_{k} u_{k i}=\left(B^{\prime \prime} H\right)^{-1}\left[P_{i}+G^{\prime} u_{i}\right] .
$$

Exploiting (4.15) in (4.14) and using (2.1), we obtain

$$
\begin{aligned}
\left(d_{i j}\right)_{j} u_{i} & =\left(B^{\prime \prime} H\right)^{-1}\left[B^{\prime \prime \prime}-\left(B^{\prime}\right)^{-1}\left(B^{\prime \prime}\right)^{2}\right]\left[P_{\ell}+G^{\prime} u_{\ell}\right] H_{\ell}+G^{\prime}\left(B^{\prime}\right)^{-1}\left[B^{\prime \prime}-B^{\prime} H^{-1}\right] \\
& =H^{-1}\left[\left(B^{\prime \prime}\right)^{-1} B^{\prime \prime \prime}-\left(B^{\prime}\right)^{-1} B^{\prime \prime}\right]\left[P_{\ell}+G^{\prime} u_{\ell}\right] H_{\ell}+G^{\prime}\left(B^{\prime}\right)^{-1}\left[B^{\prime \prime}-B^{\prime} H^{-1}\right] \\
& =H^{-1}\left[\left(B^{\prime \prime}\right)^{-1} B^{\prime \prime \prime}-\left(B^{\prime}\right)^{-1} B^{\prime \prime}\right] H_{\ell} P_{\ell}+G^{\prime}\left[\left(B^{\prime \prime}\right)^{-1} B^{\prime \prime \prime}-H^{-1}\right] .
\end{aligned}
$$

By this last equality, (4.11) becomes

$$
\begin{aligned}
\left(d_{i j} P_{i}\right)_{j}= & \left(B^{\prime \prime} H_{k}\right)_{j} a_{i j} u_{k i}-G^{\prime} H^{-1}\left[\left(B^{\prime \prime}\right)^{-1} B^{\prime \prime \prime}-\left(B^{\prime}\right)^{-1} B^{\prime \prime}\right] H_{\ell} P_{\ell} \\
& -\left(G^{\prime}\right)^{2}\left[\left(B^{\prime \prime}\right)^{-1} B^{\prime \prime \prime}-H^{-1}\right]-\left(G^{\prime}\right)^{2} H^{-1} \\
= & \left(B^{\prime \prime} H_{k}\right)_{j} a_{i j} u_{k i}-G^{\prime} H^{-1}\left[\left(B^{\prime \prime}\right)^{-1} B^{\prime \prime \prime}-\left(B^{\prime}\right)^{-1} B^{\prime \prime}\right] H_{\ell} P_{\ell}-\left(G^{\prime}\right)^{2}\left(B^{\prime \prime}\right)^{-1} B^{\prime \prime \prime} .
\end{aligned}
$$

Now, we use (4.15) to write, for any $j$ and $k$,

$$
\begin{aligned}
\left(B^{\prime \prime} H_{k}\right)_{j} & =B^{\prime \prime \prime} H_{k} H_{\ell} u_{j \ell}+B^{\prime \prime} H_{k \ell} u_{j \ell} \\
& =B^{\prime \prime \prime} H_{k}\left(B^{\prime \prime} H\right)^{-1}\left[P_{j}+G^{\prime} u_{j}\right]+B^{\prime \prime} H_{k \ell} u_{j \ell} \\
& =\left(B^{\prime \prime}\right)^{-1} B^{\prime \prime \prime} H^{-1} H_{k} P_{j}+G^{\prime}\left(B^{\prime \prime}\right)^{-1} B^{\prime \prime \prime} H^{-1} H_{k} u_{j}+B^{\prime \prime} H_{k \ell} u_{j \ell}
\end{aligned}
$$

and

$$
\begin{aligned}
a_{i j} u_{i k} & =\left[B^{\prime \prime} H_{i} H_{j}+B^{\prime} H_{i j}\right] u_{i k}=B^{\prime \prime} H_{i} H_{j} u_{i k}+B^{\prime} H_{i j} u_{i k} \\
& =B^{\prime \prime} H_{j}\left(B^{\prime \prime} H\right)^{-1}\left[P_{k}+G^{\prime} u_{k}\right]+B^{\prime} H_{i j} u_{i k} \\
& =H^{-1} H_{j} P_{k}+G^{\prime} H^{-1} H_{j} u_{k}+B^{\prime} H_{i j} u_{i k} .
\end{aligned}
$$


We put together the two formulae just obtained, getting

$$
\begin{aligned}
\left(B^{\prime \prime} H_{k}\right)_{j} a_{i j} u_{k i}= & {\left[\left(B^{\prime \prime}\right)^{-1} B^{\prime \prime \prime} H^{-1} H_{k} P_{j}+G^{\prime}\left(B^{\prime \prime}\right)^{-1} B^{\prime \prime \prime} H^{-1} H_{k} u_{j}+B^{\prime \prime} H_{k \ell} u_{j \ell}\right] } \\
& \times\left[H^{-1} H_{j} P_{k}+G^{\prime} H^{-1} H_{j} u_{k}+B^{\prime} H_{i j} u_{i k}\right] \\
= & \left(B^{\prime \prime}\right)^{-1} B^{\prime \prime \prime} H^{-2} H_{k} P_{j} H_{j} P_{k}+G^{\prime}\left(B^{\prime \prime}\right)^{-1} B^{\prime \prime \prime} H^{-2} H_{k} P_{j} H_{j} u_{k} \\
& +B^{\prime}\left(B^{\prime \prime}\right)^{-1} B^{\prime \prime \prime} H^{-1} H_{k} P_{j} H_{i j} u_{i k}+G^{\prime}\left(B^{\prime \prime}\right)^{-1} B^{\prime \prime \prime} H^{-2} H_{k} u_{j} H_{j} P_{k} \\
& +\left(G^{\prime}\right)^{2}\left(B^{\prime \prime}\right)^{-1} B^{\prime \prime \prime} H^{-2} H_{k} u_{j} H_{j} u_{k}+G^{\prime} B^{\prime}\left(B^{\prime \prime}\right)^{-1} B^{\prime \prime \prime} H^{-1} H_{k} u_{j} H_{i j} u_{i k} \\
& +B^{\prime \prime} H^{-1} H_{k \ell} u_{j \ell} H_{j} P_{k}+G^{\prime} B^{\prime \prime} H^{-1} H_{k \ell} u_{j \ell} H_{j} u_{k}+B^{\prime} B^{\prime \prime} H_{k \ell} u_{j \ell} H_{i j} u_{i k} .
\end{aligned}
$$

Making use of (4.15), (2.1) and (2.2), this becomes

$$
\begin{aligned}
\left(B^{\prime \prime} H_{k}\right)_{j} a_{i j} u_{k i}= & \left(B^{\prime \prime}\right)^{-1} B^{\prime \prime \prime} H^{-2}\left(H_{\ell} P_{\ell}\right)^{2}+G^{\prime}\left(B^{\prime \prime}\right)^{-1} B^{\prime \prime \prime} H^{-1} H_{\ell} P_{\ell} \\
& +B^{\prime}\left(B^{\prime \prime}\right)^{-1} B^{\prime \prime \prime} H^{-1} P_{j} H_{i j}\left(B^{\prime \prime} H\right)^{-1}\left[P_{i}+G^{\prime} u_{i}\right]+G^{\prime}\left(B^{\prime \prime}\right)^{-1} B^{\prime \prime \prime} H^{-1} H_{\ell} P_{\ell} \\
& +\left(G^{\prime}\right)^{2}\left(B^{\prime \prime}\right)^{-1} B^{\prime \prime \prime}+0 \\
& +B^{\prime \prime} H^{-1} H_{k \ell} P_{k}\left(B^{\prime \prime} H\right)^{-1}\left[P_{\ell}+G^{\prime} u_{\ell}\right]+0+B^{\prime} B^{\prime \prime} H_{i j} H_{k \ell} u_{i k} u_{j \ell} .
\end{aligned}
$$

Developing the products and exploiting again (2.2), we have

$$
\begin{aligned}
\left(B^{\prime \prime} H_{k}\right)_{j} a_{i j} u_{k i}= & \left(B^{\prime \prime}\right)^{-1} B^{\prime \prime \prime} H^{-2}\left(H_{\ell} P_{\ell}\right)^{2}+G^{\prime}\left(B^{\prime \prime}\right)^{-1} B^{\prime \prime \prime} H^{-1} H_{\ell} P_{\ell} \\
& +B^{\prime}\left(B^{\prime \prime}\right)^{-2} B^{\prime \prime \prime} H^{-2} H_{i j} P_{i} P_{j}+0+G^{\prime}\left(B^{\prime \prime}\right)^{-1} B^{\prime \prime \prime} H^{-1} H_{\ell} P_{\ell} \\
& +\left(G^{\prime}\right)^{2}\left(B^{\prime \prime}\right)^{-1} B^{\prime \prime \prime}+H^{-2} H_{k \ell} P_{k} P_{\ell}+0+B^{\prime} B^{\prime \prime} H_{i j} H_{k \ell} u_{i k} u_{j \ell} .
\end{aligned}
$$

Simplifying and collecting similar terms, we get

$$
\begin{aligned}
\left(B^{\prime \prime} H_{k}\right)_{j} a_{i j} u_{k i}= & \left(B^{\prime \prime}\right)^{-1} B^{\prime \prime \prime} H^{-2}\left(H_{\ell} P_{\ell}\right)^{2}+2 G^{\prime}\left(B^{\prime \prime}\right)^{-1} B^{\prime \prime \prime} H^{-1} H_{\ell} P_{\ell}+B^{\prime} B^{\prime \prime} H_{i j} H_{k \ell} u_{i k} u_{j \ell} \\
& +H^{-2}\left[B^{\prime}\left(B^{\prime \prime}\right)^{-2} B^{\prime \prime \prime}+1\right] H_{k \ell} P_{k} P_{\ell}+\left(G^{\prime}\right)^{2}\left(B^{\prime \prime}\right)^{-1} B^{\prime \prime \prime} .
\end{aligned}
$$

Plugging this into (4.16) we finally obtain

$$
\begin{aligned}
\left(d_{i j} P_{i}\right)_{j}= & \left(B^{\prime \prime}\right)^{-1} B^{\prime \prime \prime} H^{-2}\left(H_{\ell} P_{\ell}\right)^{2}+2 G^{\prime}\left(B^{\prime \prime}\right)^{-1} B^{\prime \prime \prime} H^{-1} H_{\ell} P_{\ell}+B^{\prime} B^{\prime \prime} H_{i j} H_{k \ell} u_{i k} u_{j \ell} \\
& +H^{-2}\left[B^{\prime}\left(B^{\prime \prime}\right)^{-2} B^{\prime \prime \prime}+1\right] H_{k \ell} P_{k} P_{\ell}+\left(G^{\prime}\right)^{2}\left(B^{\prime \prime}\right)^{-1} B^{\prime \prime \prime} \\
& -G^{\prime} H^{-1}\left[\left(B^{\prime \prime}\right)^{-1} B^{\prime \prime \prime}-\left(B^{\prime}\right)^{-1} B^{\prime \prime}\right] H_{\ell} P_{\ell}-\left(G^{\prime}\right)^{2}\left(B^{\prime \prime}\right)^{-1} B^{\prime \prime \prime} \\
= & \left(B^{\prime \prime}\right)^{-1} B^{\prime \prime \prime} H^{-2}\left(H_{\ell} P_{\ell}\right)^{2}+G^{\prime} H^{-1}\left[\left(B^{\prime \prime}\right)^{-1} B^{\prime \prime \prime}+\left(B^{\prime}\right)^{-1} B^{\prime \prime}\right] H_{\ell} P_{\ell} \\
& +H^{-2}\left[B^{\prime}\left(B^{\prime \prime}\right)^{-2} B^{\prime \prime \prime}+1\right] H_{k \ell} P_{k} P_{\ell}+B^{\prime} B^{\prime \prime} H_{i j} H_{k \ell} u_{i k} u_{j \ell} .
\end{aligned}
$$

The last term of the formula above coincides with the remainder $\mathscr{R}$ as defined in (4.5) and it is non-negative by (1.2) and via an application of Proposition 2.6 with $c_{i j}:=u_{i j}$. Therefore, inequality (4.4) is proved.

\section{Proof of Theorem 1.1}

The proof is similar to the one of Theorem 1 in [FV13, with the following modification. Formula (32) of [FV13] is replaced here with (4.4), on which we apply the classical Maximum Principle. 


\section{Proof of Theorem 1.2}

Up to a rotation and a translation, we may consider the origin lying in a level set $\{u=c\}$, with

$$
\nabla u(0)=|\nabla u(0)|(0, \ldots, 0,1) \neq 0 .
$$

We stress that the equation is not invariant under a rotation $R$, but the function $H$ would be replaced by $\tilde{H}:=H \circ R$. Nevertheless, the new function $\tilde{H}$ satisfies the same structural assumptions of $H$, thus we take the freedom of identifying $\tilde{H}$ with the original $H$.

We parameterize the level set of $u$ near the origin with the graph of a $C^{2}$ function $\phi$, i.e. we write $u\left(x^{\prime}, \phi\left(x^{\prime}\right)\right)=c$ for $x^{\prime} \in \mathbb{R}^{n-1}$ near the origin. By taking two derivatives, we obtain that

$$
\begin{array}{ll} 
& u_{i^{\prime}}+u_{n} \phi_{i^{\prime}}=0 \\
\text { and } \quad & u_{i^{\prime} j^{\prime}}+u_{i^{\prime} n} \phi_{j^{\prime}}+u_{j^{\prime} n} \phi_{i^{\prime}}+u_{n n} \phi_{i^{\prime}} \phi_{j^{\prime}}+u_{n} \phi_{i^{\prime} j^{\prime}}=0,
\end{array}
$$

for any $i^{\prime}, j^{\prime} \in\{1, \ldots, n-1\}$, where the derivatives of $u$ are evaluated at $\left(x^{\prime}, \phi\left(x^{\prime}\right)\right)$ and the derivatives of $\phi$ are evaluated at $x^{\prime}$. In particular, by taking $x^{\prime}=0$, we obtain that $\phi_{i^{\prime}}(0)=0$ and $u_{i^{\prime} j^{\prime}}(0)=-u_{n}(0) \phi_{i^{\prime} j^{\prime}}(0)$, for any $i^{\prime}, j^{\prime} \in\{1, \ldots, n-1\}$.

Consequently, we have that all the principal curvatures of the level set at 0 vanish if and only if $\phi_{i^{\prime} j^{\prime}}(0)=0$ for any $i^{\prime}, j^{\prime} \in\{1, \ldots, n-1\}$, and so, by (6.1), if and only if

$$
u_{i^{\prime} j^{\prime}}(0)=0 \text { for any } i^{\prime}, j^{\prime} \in\{1, \ldots, n-1\} .
$$

Hence, we establish (6.2) in order to complete the proof of Theorem 1.2, The proof of (6.2) is based on Proposition 2.6. We need to check that the hypotheses of Proposition 2.6 are satisfied in this case. First of all, we have that (2.7) is guaranteed by (6.1) (here $\xi=\nabla u(0)$ ). Then, by Theorem 1.1, we know that (1.11) holds true in the whole connected component $\mathscr{S}$ that contains 0 . As a consequence, $P$ vanishes identically in $\mathscr{S}$, thus we obtain from (4.4) and (4.5) that

$$
0=\left(d_{i j} P_{i}\right)_{j}-b_{k} P_{k}=\mathscr{R}=B^{\prime} B^{\prime \prime} H_{i j} H_{k \ell} u_{i k} u_{j \ell} .
$$

This says that equality holds in (2.6) with $\xi=\nabla u(0)$ and $c_{i j}=u_{i j}$. Accordingly, the hypotheses of Proposition 2.6 are fulfilled and we obtain (6.2) from (2.8). The proof of Theorem 1.2 is therefore complete.

\section{Proof of Theorem 1.3}

In this case $u$ is of class $C^{3}$ everywhere, due to Proposition 3.2, therefore we can differentiate (1.4) and write it in non-divergence form as

$$
(B \circ H)_{i j} u_{i j}+F^{\prime}(u)=0 .
$$

Notice that the matrix $\left\{(B \circ H)_{i j}\right\}_{i, j \in\{1, \ldots, n\}}$ is elliptic since, by assumption (B),

$$
(B \circ H)_{i j} \xi_{i} \xi_{j} \geqslant \gamma|\xi|^{2} .
$$

Moreover, by Lemma 2.9, $B$ is of class $C^{2}$ at the origin with

$$
B^{\prime \prime}(0)>0 \text {. }
$$

Notice in particular that the last inequality follows combining (2.22) and (7.1).

Now, we observe that, in view of Theorem 1.2, $u$ is one-dimensional and that its profile $u_{0}$ satisfies the ordinary differential equation (1.12) on an interval. Also recall that $u$, and consequently $u_{0}$, is bounded, with bounded gradient.

Thanks to (7.2), the linearized equation can be represented as a first order system of ODEs in canonical form, so that $u_{0}$ extends to a global solution $\hat{u}_{0}$ by the standard theory for Cauchy problems with globally Lipschitz nonlinearities (see e.g. page 146 in [PSV84]).

Finally, by the Unique Continuation Principle (see e.g. [H05]), we have that $u$ agrees everywhere with the one-dimensional extension of $\hat{u}_{0}$ to $\mathbb{R}^{n}$. This concludes the proof. 


\section{Proof of Theorem 1.4}

Assume $\mathscr{S}$ to be any connected component of $\{\nabla u \neq 0\}$. We claim that

$\mathscr{S}$ is foliated by level sets of $u$ which are union of parallel affine hyperplanes and so $\partial \mathscr{S}$ is the union of (at most two) parallel hyperplanes.

In order to prove this, fix $x_{\star} \in \mathscr{S}$ and consider the level set $S_{x_{\star}}:=\left\{u=u\left(x_{\star}\right)\right\}$. Notice that

$$
S_{x_{\star}} \subseteq\{\nabla u \neq 0\} .
$$

Indeed, if $x \in S_{x_{\star}}$, then from (1.11) we deduce that

$$
\begin{aligned}
& B^{\prime}(H(\nabla u(x))) H(\nabla u(x))-B(H(\nabla u(x)))=c_{u}-F(u(x)) \\
& =c_{u}-F\left(u\left(x_{\star}\right)\right)=B^{\prime}\left(H\left(\nabla u\left(x_{\star}\right)\right)\right) H\left(\nabla u\left(x_{\star}\right)\right)-B\left(H\left(\nabla u\left(x_{\star}\right)\right)\right)>0,
\end{aligned}
$$

because $x_{\star}$ is in $\{\nabla u \neq 0\}$ and so we can apply (2.15) taking $t:=H\left(\nabla u\left(x_{\star}\right)\right)>0$. But then, also $x \in\{\nabla u \neq 0\}$, since otherwise $H(\nabla u)$ would vanish, in contradiction with (8.3). This establishes (8.2) so that we are allowed to apply Theorem 1.2, concluding that every connected component of $S_{x_{\star}}$ is contained in a hyperplane, say $\ell_{x_{\star}}$. In particular, we point out that

$$
\text { the connected component of } S_{x_{\star}} \text { which contains } x_{\star} \text { is equal to } \ell_{x_{\star}} \text {. }
$$

Indeed, $S_{x_{\star}}$ is closed in the relative topology of $\ell_{x_{\star}}$, being $u$ continuous. Furthermore, $S_{x_{\star}}$ is also relatively open, by (8.2) and applying Theorem 1.2 together with the Implicit Function Theorem. Thus, (8.4) holds true.

Combining (8.4) and (8.2) we immediately obtain (8.1).

Let now $\omega$ denote a vector normal to all the hyperplanes in (8.1). We claim that

$$
u\left(x_{0}\right)=u\left(y_{0}\right) \text { if }\left(x_{0}-y_{0}\right) \cdot \omega=0 .
$$

To check this, fix $x_{0} \in \mathbb{R}^{n}$. If $\nabla u=0$ on the whole $\ell_{x_{0}}$, then (8.5) follows from the Fundamental Theorem of Calculus. Conversely, let $x_{\sharp}$ be a point in $\ell_{x_{0}} \cap\{\nabla u \neq 0\}$. By (8.1) (applied to $x_{\sharp}$ ), we have that $u$ is constant on $\ell_{x_{\sharp}}$, which, in turn, is equal to $\ell_{x_{0}}$. Thus, (8.5) is proved and so is the desired one-dimensional Euclidean symmetry.

\section{Proof of Theorem 1.5}

Let $r:=u\left(x_{0}\right)$ and fix a point $x \in \mathbb{R}^{n} \backslash\left\{x_{0}\right\}$. In order to establish the thesis of Theorem 1.5. we shall show that $u(x)=r$. Consider the $C^{1}$ function $\varphi$, defined by setting

$$
\varphi(t):=u\left(t x+(1-t) x_{0}\right)-r \text { for any } t \in[0,1] .
$$

In the following we will sometimes adopt the short notation $x_{t}:=t x+(1-t) x_{0}$. Notice that, by the regularity of $u$, the function $t \mapsto\left|\nabla u\left(t x+(1-t) x_{0}\right)\right|$ is bounded on $[0,1]$. We may therefore apply Lemma 2.8 (and also recall the notation there introduced) to compute

$$
\begin{aligned}
|\dot{\varphi}(t)|^{p^{*}} & \leqslant\left|x-x_{0}\right|^{p^{*}}\left|\nabla u\left(t x+(1-t) x_{0}\right)\right|^{p^{*}} \\
& =\left|x-x_{0}\right|^{p^{*}}\left|\nabla u\left(x_{t}\right)\right|^{p^{*}} \\
& \leqslant \frac{\left|x-x_{0}\right|^{p^{*}}}{h^{p^{*}}} H^{p^{*}}\left(\nabla u\left(x_{t}\right)\right) \\
& \leqslant \frac{\left|x-x_{0}\right|^{p^{*}}}{\varepsilon h^{p^{*}}}\left[B^{\prime}\left(H\left(\nabla u\left(x_{t}\right)\right) H\left(\nabla u\left(x_{t}\right)\right)-B\left(H\left(\nabla u\left(x_{t}\right)\right)\right)\right],\right.
\end{aligned}
$$


for some $\varepsilon>0$. Next, recalling (1.9) and the assumptions of Theorem 1.5, we have that

$$
\begin{aligned}
|\dot{\varphi}(t)|^{p^{*}} & \leqslant \frac{\left|x-x_{0}\right|^{p^{*}}}{\varepsilon h^{p^{*}}}\left[c_{u}-F\left(u\left(x_{t}\right)\right)\right] \\
& =\frac{\left|x-x_{0}\right|^{p^{*}}}{\varepsilon h^{p^{*}}}\left[F(r)-F\left(u\left(t x+(1-t) x_{0}\right)\right)\right] \\
& =-\frac{\left|x-x_{0}\right|^{p^{*}}}{\varepsilon h^{p^{*}}} \int_{r}^{u\left(t x+(1-t) x_{0}\right)} F^{\prime}(\sigma) d \sigma .
\end{aligned}
$$

Then, we employ alternatively the Lipschitz regularity of $F^{\prime}$ or (1.13) to write

$$
\left|F^{\prime}(\sigma)\right| \leqslant c|r-\sigma|^{p^{*}-1} \text { for any } \sigma \in\left[\inf _{\mathbb{R}^{n}} u, \sup _{\mathbb{R}^{n}} u\right],
$$

for some positive constant $c$. Using this estimate in (9.1), we get

$$
\begin{aligned}
|\dot{\varphi}(t)|^{p^{*}} & \leqslant \frac{c\left|x-x_{0}\right|^{p^{*}}}{\varepsilon h^{p^{*}}}\left|\int_{r}^{u\left(t x+(1-t) x_{0}\right)}\right| r-\left.\sigma\right|^{p^{*}-1} d \sigma \mid \\
& =\frac{c\left|x-x_{0}\right|^{p^{*}}}{\varepsilon p^{*} h^{p^{*}}}\left|u\left(t x+(1-t) x_{0}\right)-\sigma\right|^{p^{*}} \\
& =\frac{c\left|x-x_{0}\right|^{p^{*}}}{\varepsilon p^{*} h^{p^{*}}}|\varphi(t)|^{p^{*}}
\end{aligned}
$$

which yields, if $\varphi(t) \neq 0$,

$$
\left|\frac{\dot{\varphi}(t)}{\varphi(t)}\right| \leqslant \frac{c^{1 / p^{*}}\left|x-x_{0}\right|}{\varepsilon p^{* 1 / p^{*}} h}=: K .
$$

Finally, set $\psi(t):=(\varphi(t))^{2} e^{-K t}$, for any $t \in[0,1]$. Differentiating $\psi$, we obtain

$$
\begin{aligned}
\dot{\psi}(t) & =\varphi(t) e^{-K t}[\dot{\varphi}(t)-K \varphi(t)] \\
& =\left\{\begin{array}{cc}
(\varphi(t))^{2} e^{-K t}\left[\frac{\dot{\varphi}(t)}{\varphi(t)}-K\right] & \text { if } \varphi(t) \neq 0 \\
0 & \text { if } \varphi(t)=0
\end{array}\right. \\
& \leqslant 0,
\end{aligned}
$$

so that $\psi$ is non-increasing. Hence

$$
(u(x)-r)^{2} e^{-K}=\varphi(1)^{2} e^{-K}=\psi(1) \leqslant \psi(0)=\varphi(0)^{2}=\left(u\left(x_{0}\right)-r\right)^{2}=0,
$$

and therefore $u(x)=r$, which concludes the proof.

\section{Proof of Theorem 1.6}

We will suppose, without loss of generality, that

$$
u \text { is not constant. }
$$

Then, assume by contradiction that there exists $r_{0} \in\left(\inf _{\mathbb{R}^{n}} u, \sup _{\mathbb{R}^{n}} u\right)$ such that

$$
\sup \left\{F(r): r \in\left[\inf _{\mathbb{R}^{n}} u, \sup _{\mathbb{R}^{n}} u\right]\right\}=c_{u}=F\left(r_{0}\right) .
$$

By the continuity of $u$, there also exists a point $x_{0} \in \mathbb{R}^{n}$ such that $u\left(x_{0}\right)=r_{0}$. Moreover, $r_{0}$ is a local maximum for $F$, so that $F^{\prime}\left(r_{0}\right)=0$. Thus, we can apply Theorem 1.5, deducing that $u$ is constant, in contradiction to (10.1). 


\section{Appendix A. An EXAmple in Which $H$ is NOT A NORM}

Here we present an example in which $H$ satisfies the structural assumptions requested in this paper without being a norm. Indeed $H$ will be positive homogeneous of degree 1 , but not necessarily a norm. More precisely, given any convex set $\mathscr{K}$ described as a graph over the sphere by

$$
\mathscr{K}:=\left\{\frac{t \xi}{\Theta(\xi)}, \xi \in S^{n-1}, t \in[0,1]\right\},
$$

with $\Theta \in C_{\text {loc }}^{3, \beta}\left(\mathbb{R}^{n} \backslash\{0\},(0,+\infty)\right) \cap L_{\text {loc }}^{\infty}\left(\mathbb{R}^{n}\right)$, and with

(A.1) principal curvatures along $\partial \mathscr{K}$ bounded from below by some $c>0$, we construct an admissible $H$ for which

$$
\{H=1\}=\partial \mathscr{K} .
$$

Precisely, such $H$ is defined, for any $\xi \in \mathbb{R}^{n} \backslash\{0\}$, by

$$
H(\xi):=|\xi| \Theta\left(\frac{\xi}{|\xi|}\right) .
$$

Notice that $H$ is not even, unless so is $\Theta$. Therefore, in general, $H$ is not a norm. We have that

$$
\begin{aligned}
\partial \mathscr{K} & =\left\{\zeta \in \mathbb{R}^{n}: \zeta=\frac{\xi}{\Theta(\xi)}, \xi \in S^{n-1}\right\} \\
& =\left\{\zeta \in \mathbb{R}^{n}:|\zeta|=\frac{1}{\Theta(\xi)}, \xi=\frac{\zeta}{|\zeta|} \in S^{n-1}\right\} \\
& =\left\{\zeta \in \mathbb{R}^{n}: H(\zeta)=1\right\},
\end{aligned}
$$

that is (A.2).

Our goal is to show that

$$
\text { if } B(t)=\frac{t^{p}}{p} \text { with } p>1 \text {, then assumption (A) holds. }
$$

To this end, we first notice that

$$
H \text { is positively homogeneous of degree } 1 \text {, }
$$

and so the range of Hess $(H)(\xi)$ lies in $\xi^{\perp}$, thanks to point (ii) in Lemma 2.2. Then we show that

$$
\text { Hess }(H)(\xi) \text { is a positive definite endomorphism on } \xi^{\perp} \text {. }
$$

To see this, we make the relation between the second fundamental form of $\partial \mathscr{K}$ and the Hessian of $H$ explicit. Although we believe this fact to be well-known to the experts, we still provide all the details. Let $\xi \in \mathbb{R}^{n} \backslash\{0\}$ with $H(\xi)=1$ and $v, w \in T_{\xi}(\partial \mathscr{K})$. By indicating with $\nu=\left(\nu_{1}, \ldots, \nu_{n}\right)$ the interior normal of $\partial \mathscr{K}$ at $\xi$, we obtain that the second fundamental form of $\partial \mathscr{K}$ at $\xi$ applied to $v$ and $w$ equals

$$
\mathbb{I}_{\xi}(v, w)=-\left\langle d_{\xi} \nu(v), w\right\rangle=-\frac{\partial \nu_{j}}{\partial \xi_{i}} v_{i} w_{j}
$$

Since $\nu(\xi)=-\nabla H(\xi) /|\nabla H(\xi)|$, we compute

$$
\frac{\partial \nu_{j}}{\partial \xi_{i}}=-\frac{H_{i j}(\xi)}{|\nabla H(\xi)|}+\frac{H_{i}(\xi) H_{j}(\xi)}{|\nabla H(\xi)|^{3}} .
$$

Being by definition $v, w \perp \nabla H(\xi)$, we obtain

$$
\mathbb{I}_{\xi}(v, w)=\frac{H_{i j}(\xi)}{|\nabla H(\xi)|} v_{i} w_{j}-\frac{H_{i}(\xi) H_{j}(\xi)}{|\nabla H(\xi)|^{3}} v_{i} w_{j}=\frac{H_{i j}(\xi) v_{i} w_{j}}{|\nabla H(\xi)|} .
$$


Furthermore, by (A.3)

$$
|\xi|=\frac{H(\xi)}{\Theta\left(\frac{\xi}{|\xi|}\right)}=\frac{1}{\Theta\left(\frac{\xi}{|\xi|}\right)} \leqslant\left[\min _{\zeta \in S^{n-1}} \Theta(\zeta)\right]^{-1}=: c^{\prime},
$$

and so, using (A.5)

$$
|\nabla H(\xi)| \geqslant \frac{1}{|\xi|} \nabla H(\xi) \cdot \xi=\frac{H(\xi)}{|\xi|}=\frac{1}{|\xi|} \geqslant \frac{1}{c^{\prime}},
$$

Therefore, by (A.1) and (A.7) we conclude that, for any $\xi \in \mathbb{R}^{n} \backslash\{0\}$ with $H(\xi)=1$ and any $v \in \nabla H(\xi)^{\perp}$,

$$
H_{i j}(\xi) v_{i} v_{j}=|\nabla H(\xi)| \mathbb{\Pi}_{\xi}(v, v) \geqslant \frac{c}{c^{\prime}}|v|^{2} .
$$

Now, by homogeneity we extend the previous estimate to any $\xi \in \mathbb{R}^{n} \backslash\{0\}$. For this, fixed any $\xi \in \mathbb{R}^{n} \backslash\{0\}$ and any $v \in \nabla H(\xi)^{\perp}$, we define $\tilde{\xi}:=\xi / H(\xi)$. By (A.5), we have that $H(\tilde{\xi})=1$ and that $\nabla H(\tilde{\xi})=\nabla H(\xi)$. Hence we can apply (A.8) to $\tilde{\xi}$ and $v \in \nabla H(\tilde{\xi})^{\perp}$, and once more the homogeneity in (A.5), obtaining

$$
H_{i j}(\xi) v_{i} v_{j}=H_{i j}(H(\xi) \tilde{\xi}) v_{i} v_{j}=(H(\xi))^{-1} H_{i j}(\tilde{\xi}) v_{i} v_{j} \geqslant \frac{c}{c^{\prime}}(H(\xi))^{-1}|v|^{2} .
$$

On the other hand,

$$
H(\xi) \in\left[|\xi| \min _{\zeta \in S^{n-1}} \Theta(\zeta),|\xi| \max _{\zeta \in S^{n-1}} \Theta(\zeta)\right]
$$

This and (A.9) imply that

$$
H_{i j}(\xi) v_{i} v_{j} \geqslant \tilde{c}|\xi|^{-1}|v|^{2},
$$

for some $\tilde{c}>0$. We now complete the proof of (A.6). We observe that it is enough to prove that, for any $\xi, \eta \in S^{n-1}$, with $\eta \in \xi^{\perp}$,

$$
H_{i j}(\xi) \eta_{i} \eta_{j} \geqslant \tilde{c}
$$

Since $\xi$ is not orthogonal to $\nabla H(\xi)$, we can write $\eta=\alpha \xi+v$, for some $\alpha \in \mathbb{R}$ and $v \perp \nabla H(\xi)$. On the other hand, being $\eta$ and $\xi$ orthogonal, by Pythagoras' theorem we have that

$$
|v|^{2}=|\eta|^{2}+\alpha^{2}|\xi|^{2}=1+\alpha^{2} \geqslant 1 \text {. }
$$

Therefore, by (2.2), (A.10) and (A.12) we are able to conclude that

$$
H_{i j}(\xi) \eta_{i} \eta_{j}=H_{i j}(\xi)\left(\alpha \xi_{i}+v_{i}\right)\left(\alpha \xi_{j}+v_{j}\right)=H_{i j}(\xi) v_{i} v_{j} \geqslant \tilde{c}|v|^{2} \geqslant \tilde{c}
$$

which is (A.11).

Now we point out that

$$
\begin{aligned}
{[\operatorname{Hess}(B \circ H)(\xi)]_{i j} \zeta_{i} \zeta_{j} } & =\left[B^{\prime \prime}(H(\xi)) H_{i}(\xi) H_{j}(\xi)+B^{\prime}(H(\xi)) H_{i j}(\xi)\right] \zeta_{i} \zeta_{j} \\
& =(p-1)(H(\xi))^{p-2}\left(H_{i}(\xi) \zeta_{i}\right)^{2}+(H(\xi))^{p-1} H_{i j}(\xi) \zeta_{i} \zeta_{j} .
\end{aligned}
$$

Moreover, by homogeneity, $H(\xi) \in\left[c_{1}|\xi|, C_{1}|\xi|\right],|\nabla H(\xi)| \leqslant C_{1}$, and $\left|H_{i j}(\xi)\right| \leqslant C_{1} /|\xi|$, for suitable $C_{1} \geqslant c_{1}>0$. Therefore

$$
[\operatorname{Hess}(B \circ H)(\xi)]_{i j} \zeta_{i} \zeta_{j} \leqslant(p-1) C_{1}^{p}|\xi|^{p-2}|\zeta|^{2}+C_{1}^{p}|\xi|^{p-2}|\zeta|^{2} .
$$

Now we claim that

$$
[\operatorname{Hess}(B \circ H)(\xi)]_{i j} \zeta_{i} \zeta_{j} \geqslant c_{\star}|\xi|^{p-2}|\zeta|^{2},
$$

for some $c_{\star}>0$. To prove it, we observe that $B \circ H$ is homogeneous of degree $p$, hence Hess $(B \circ H)$ is homogeneous of degree $p-2$, so without loss of generality we may assume $|\xi|=1$. Also, we write $\zeta=\alpha \xi+w$, with $\alpha \in \mathbb{R}$ and $w \in \xi^{\perp}$, so that $|\zeta|^{2}=\alpha^{2}+|w|^{2}$. We observe that

$$
(H(\xi))^{p-1} H_{i j}(\xi) \zeta_{i} \zeta_{j}=(H(\xi))^{p-1} H_{i j}(\xi) w_{i} w_{j} \geqslant c_{1}^{p-1} c|w|^{2},
$$


due to point (ii) in Lemma 2.2 and (A.6). Now, we distinguish two cases. If

$$
|w| \geqslant c_{1}|\zeta| /\left(2 C_{1}+2 c_{1}\right)
$$

then we use (A.13) and (A.16), to obtain

$$
[\operatorname{Hess}(B \circ H)(\xi)]_{i j} \zeta_{i} \zeta_{j} \geqslant(H(\xi))^{p-1} H_{i j}(\xi) \zeta_{i} \zeta_{j} \geqslant \frac{c_{1}^{p+1} c}{4\left(C_{1}+c_{1}\right)^{2}}|\zeta|^{2} .
$$

This proves (A.15) in this case. On the other hand, if

$$
|w|<c_{1}|\zeta| /\left(2 C_{1}+2 c_{1}\right) \leqslant \min \left\{c_{1}|\zeta| /\left(2 C_{1}\right),|\zeta| / 2\right\},
$$

we recall point $(i)$ in Lemma 2.2 and we see that

$$
\begin{aligned}
\left|H_{i}(\xi) \zeta_{i}\right| & \geqslant\left|\alpha H_{i}(\xi) \xi_{i}\right|-\left|H_{i}(\xi) w_{i}\right| \geqslant|\alpha H(\xi)|-C_{1}|w| \geqslant c_{1}|\alpha|-C_{1}|w| \\
& =c_{1} \sqrt{|\zeta|^{2}-|w|^{2}}-C_{1}|w| \geqslant c_{1} \sqrt{\frac{3}{4}}|\zeta|-c_{1} \frac{|\zeta|}{2}=\frac{c_{1}(\sqrt{3}-1)}{2}|\zeta| .
\end{aligned}
$$

Thus, by (A.13) and (A.16),

$$
\begin{aligned}
{[\operatorname{Hess}(B \circ H)(\xi)]_{i j} \zeta_{i} \zeta_{j} } & \geqslant(p-1)(H(\xi))^{p-2}\left(H_{i}(\xi) \zeta_{i}\right)^{2} \\
& \geqslant \frac{(p-1) c_{1}^{p}(\sqrt{3}-1)^{2}}{4}|\zeta|^{2} .
\end{aligned}
$$

This completes the proof of (A.15). Then (A.14) and (A.15) establish (A.4) in this case.

\section{Appendix B. A characterization of $H$ under assumption (B)}

We present here an exhaustive characterization of the functions $H$ that satisfy (B). Indeed, we prove that in this case $H$ is as in (1.8).

Let $H$ and $B$ be as in hypothesis (B). Notice that, as showed in Section 7 we have that $B \in C^{2}([0,+\infty))$, with

$$
B^{\prime \prime}(0)>0 \text {. }
$$

By Lemma 2.3, we know that

$$
\partial_{i}(B \circ H)(\xi)= \begin{cases}B^{\prime}(H(\xi)) H_{i}(\xi) & \text { if } \xi \neq 0 \\ 0 & \text { if } \xi=0 .\end{cases}
$$

Thus, we can proceed to compute the second partial derivatives of $B \circ H$ at the origin. Writing as $e_{j}$ the $j$-th component of the standard basis of $\mathbb{R}^{n}$, we get

$$
\begin{aligned}
\partial_{i j}^{2}(B \circ H)(0) & =\lim _{t \rightarrow 0} \frac{B^{\prime}\left(H\left(t e_{j}\right)\right) H_{i}\left(t e_{j}\right)-0}{t}=\lim _{t \rightarrow 0^{ \pm}} \frac{B^{\prime}\left(|t| H\left( \pm e_{j}\right)\right) H_{i}\left( \pm e_{j}\right)}{t} \\
& = \pm \lim _{t \rightarrow 0^{ \pm}} \frac{B^{\prime}\left(|t| H\left( \pm e_{j}\right)\right)}{|t| H\left( \pm e_{j}\right)} H_{i}\left( \pm e_{j}\right) H\left( \pm e_{j}\right)= \pm B^{\prime \prime}(0) H_{i}\left( \pm e_{j}\right) H\left( \pm e_{j}\right) .
\end{aligned}
$$

Therefore, recalling (B.1), we may conclude that the limit exists if and only if

$$
H_{i}\left(e_{j}\right) H\left(e_{j}\right)=-H_{i}\left(-e_{j}\right) H\left(-e_{j}\right), \quad \text { for any } i, j \in\{1, \ldots, n\},
$$

or, equivalently,

$$
\partial_{i}\left(H^{2}\right)\left(e_{j}\right)=-\partial_{i}\left(H^{2}\right)\left(-e_{j}\right), \quad \text { for any } i, j \in\{1, \ldots, n\} .
$$

Knowing this, we can check the continuity of the derivatives at the origin. Since

$$
\partial_{i j}^{2}(B \circ H)(\xi)=B^{\prime \prime}(H(\xi)) H_{i}(\xi) H_{j}(\xi)+B^{\prime}(H(\xi)) H_{i j}(\xi),
$$


for any $\xi \neq 0$, we compute

$$
\begin{aligned}
\lim _{\xi \rightarrow 0} \partial_{i j}^{2}(B \circ H)(\xi)= & \lim _{\xi \rightarrow 0}\left[B^{\prime \prime}(H(\xi))-\frac{B^{\prime}(H(\xi))}{H(\xi)}\right] H_{i}(\xi) H_{j}(\xi) \\
& +\lim _{\xi \rightarrow 0} \frac{B^{\prime}(H(\xi))}{H(\xi)}\left[H_{i}(\xi) H_{j}(\xi)+H(\xi) H_{i j}(\xi)\right] \\
= & : L_{1}+L_{2} .
\end{aligned}
$$

We observe that $L_{1}=0$, since $H_{i} H_{j}$ is homogeneous of degree 0 , and thus bounded, and $B$ is of class $C^{2}$ at the origin. Therefore we get

$$
\lim _{\xi \rightarrow 0} \partial_{i j}^{2}(B \circ H)(\xi)=L_{2}=B^{\prime \prime}(0) \lim _{\xi \rightarrow 0}\left[H_{i}(\xi) H_{j}(\xi)+H(\xi) H_{i j}(\xi)\right],
$$

so that, recalling (B.2), the continuity of the second derivatives is equivalent to

$$
\lim _{\xi \rightarrow 0}\left[H_{i}(\xi) H_{j}(\xi)+H(\xi) H_{i j}(\xi)\right]=H_{i}\left(e_{j}\right) H\left(e_{j}\right) .
$$

Rewriting last identity as

$$
\lim _{\xi \rightarrow 0} \partial_{i j}^{2}\left(\frac{H^{2}}{2}\right)(\xi)=\partial_{i}\left(\frac{H^{2}}{2}\right)\left(e_{j}\right)
$$

we notice that, since $\partial_{i j}^{2}\left(H^{2} / 2\right)$ is a homogeneous function of degree 0 , by (B.4) it has limit at the origin and so it is necessarily constant. This means that $H^{2}$ is a polynomial of degree 2 and thus

$$
H(\xi)=H_{M}(\xi):=\sqrt{\langle M \xi, \xi\rangle}, \quad \text { for any } \xi \in \mathbb{R}^{n},
$$

with $M \in \operatorname{Mat}_{n}(\mathbb{R})$ symmetric and positive definite. The function $H_{M}$ thus defined is clearly positive homogeneous of degree 1 and it satisfies (B.3), since it is even.

\section{Appendix C. The Wulff Shape: a Physical interpretation}

The convex anisotropy $H$ we dealt with all along the present work is widely considered in the literature. A particular set is typically related to it: the Wulff shape. Considering the dual function $H^{*}$ of $H$, defined by setting

$$
H^{*}(x):=\sup _{|\xi|=1} \frac{\langle\xi, x\rangle}{H(\xi)}, \quad \text { for all } x \in \mathbb{R}^{n},
$$

the Wulff shape $W_{H}$ of $H$ is the 1 -sublevel set of $H^{*}$, that is

$$
W_{H}:=\left\{x \in \mathbb{R}^{n}: H^{*}(x) \leqslant 1\right\} .
$$

As shown by the classical Wulff theorem (see e.g. Theorem 1.1 in [T78]), $W_{H}$ is the set which minimizes the anisotropic interfacial energy

$$
\Omega \longmapsto \int_{\partial \Omega} H(\nu(x)) d \mathcal{H}^{n-1}(x),
$$

between all sets $\Omega$ having the same prescribed volume.

This property is frequently used, for instance, to deduce the equilibrium shape of a crystal, due to the anisotropic nature of the forces there involved. A less common application is described in [T78, Section 2], where the author addresses the problem of determining the closed path a trawler should follow in order to enclose a fixed amount of fish in the shortest time. Assuming the fish to be uniformly distributed in the sea and denoting by $H(\xi)$ the time the sailboat employs to travel, say, one mile in direction $\xi \in \partial B_{1}$, it is proved that the optimal path is given by following the frontier of a suitable dilation of the Wulff shape of $H$.

Next we present another physical interpretation of the Wulff shape, which arises quite naturally in a dynamical model related to our framework. Consider equations (1.4) and (1.12) 
in the case $B(t)=t^{2} / 2$, with no forcing terms (i.e. when $F:=0$ ) and take the corresponding hyperbolic evolutionary equation

$$
u_{t t}=\operatorname{div}(H(\nabla u) \nabla H(\nabla u)) .
$$

Notice that (C.2) is the classical wave equation when $H(\xi):=|\xi|$. Then, define $u^{\omega}$ to be a one-dimensional travelling wave of velocity $c_{\omega}>0$, that is

$$
u^{\omega}(x, t):=u_{0}\left(\omega \cdot x-c_{\omega} t\right)
$$

with $u_{0}$ smooth and increasing for simplicity. Using the homogeneity properties of $H$ (e.g. (2.1) and (2.2)), we see that, if $u_{0}$ is not affine, then it is a solution of (C.2) if and only if

$$
c_{\omega}=H(\omega) .
$$

In this setting, the points reached by the plane wave $u^{\omega}$ in a unit of time form exactly the set $\left\{x \in \mathbb{R}^{n}: \omega \cdot x \leqslant H(\omega)\right\}$. By taking all the possible directions $\omega \in \partial B_{1}$ we obtain

$$
\bigcap_{\omega \in \partial B_{1}}\left\{x \in \mathbb{R}^{n}: \omega \cdot x \leqslant H(\omega)\right\}
$$

which is the Wulff shape of the velocity function $\omega \mapsto c_{\omega}=H(\omega)$, as one can easily check recalling definition (C.1).

\section{ACKNOWLEDGEMENTS}

We thank Guglielmo Albanese and Simona Scoleri for several interesting conversations. This work was supported by the ERC Grant $\epsilon$ (Elliptic Pde's and Symmetry of Interfaces and Layers for Odd Nonlinearities).

\section{REFERENCES}

[AC77] E. Arbel, J.W. Cahn, A method for the absolute measurement of anisotropic surface free energies, Surf. Sci., 66:14-24, 1977.

[BNP01a] G. Bellettini, M. Novaga, M. Paolini, Characterization of facet breaking for non-smooth mean curvature flow in the convex case, Interfaces and Free Bound., 3:415-446, 2001.

[BNP01b] G. Bellettini, M. Novaga, M. Paolini, On a crystalline variational problem, part I: first variation and global $L^{\infty}$ regularity, Arch. Ration. Mech. Anal., 157:165-191, 2001.

[CGS94] L. Caffarelli, N. Garofalo, F. Segàla, A gradient bound for entire solutions of quasi-linear equations and its consequences, Comm. Pure Appl. Math., 47:1457-1473, 1994.

[CFV12] D. Castellaneta, A. Farina, E. Valdinoci, A pointwise gradient estimate for solutions of singular and degenerate PDE's in possibly unbounded domains with nonnegative mean curvature, Commun. Pure Appl. Anal., 11:1983-2003, 2012.

[C84] A.A. Chernov, Modern Crystallography III. Crystal Growth, Springer, Berlin, 1984.

[CS09] A. Cianchi, P. Salani, Overdetermined anisotropic elliptic problems, Math. Ann., 345:859-881, 2009.

[C04] U. Clarenz, The Wulff shape minimizes an anisotropic Willmore functional, Interfaces Free Bound., 6:351-359, 2004.

[DiB83] E. Di Benedetto, $C^{1+\alpha}$ local regularity of weak solutions of degenerate elliptic equations, Nonlinear Anal., 7:827-850, 1983.

[D44] A. Dinghas, Über einen geometrischen Satz von Wulff für die Gleichgewichtsform von Kristallen, Z. Kristallogr., 105:304-314, 1944.

[EO04] S. Esedoḡlu, S.J. Osher, Decomposition of images by the anisotropic Rudin-Osher-Fatemi model, Commun. Pure Appl. Math., 57:1609-1626, 2004.

[FSV08] A. Farina, B. Sciunzi, E. Valdinoci, Bernstein and De Giorgi type problems: new results via a geometric approach, Ann. Sc. Norm. Super. Pisa Cl. Sci. (5), 7:741-791, 2008.

[FV10] A. Farina, E. Valdinoci, A pointwise gradient estimate in possibly unbounded domains with nonnegative mean curvature, Adv. Math., 225:2808-2827, 2010.

[FV13] A. Farina, E. Valdinoci, Gradient bounds for anisotropic partial differential equations, Calc. Var. Partial Differential Equations, DOI 10.1007/s00526-013-0605-9, 2013. 
[FM91] I. Fonseca, S. Müller, A uniqueness proof for the Wulff theorem, Proc. Roy. Soc. Edinburgh Sect. A, 119:125-136, 1991.

[G06] Y. Giga, Surface evolution equations. A level set approach, Birkhäuser Verlag, Basel, 2006.

[G93] M.E. Gurtin, Thermomechanics of evolving phase boundaries in the plane, Oxford University Press, New York, 1993.

[H05] L. Hörmander, The analysis of linear partial differential operators II. Differential operators with constant coefficients, Reprint of the 1983 original, Springer, Berlin, 2005.

[LU68] O.A. Ladyzhenskaya, N.N. Uraltseva, Linear and Quasilinear Elliptic Equations, Academic Press, New York, 1968.

[M85] L. Modica, A gradient bound and a Liouville theorem for nonlinear Poisson equations, Comm. Pure Appl. Math., 38:679-684, 1985.

[MBK77] H. Müller-Krumbhaar, T.W. Burkhardt, D.M. Kroll, A generalized kinetic equation for crystal growth, J. Crystal Growth, 38:13-22, 1977.

[NP99] M. Novaga, E. Paolini, A computational approach to fractures in crystal growth, Atti Accad. Naz. Lincei Cl. Sci. Fis. Mat. Natur., 10:47-56, 1999.

[OBGXY05] S. Osher, M. Burger, D. Goldfarb, J. Xu, W. Yin, An iterative regularization method for total variation-based image restoration, Multiscale Model. Simul., 4:460-489, 2005.

[P76] L.E. Payne, Some remarks on maximum principles, J. Anal. Math., 30:421-433, 1976.

[PSV84] L. C. Piccinini, G. Stampacchia, and G. Vidossich, Ordinary differential equations in $\mathbb{R}^{n}$. Problems and methods, Springer-Verlag, New York, 1984.

[S81] R.P. Sperb, Maximum Principles and Their Applications Academic Press, New York, 1981.

[T78] J.E. Taylor, Crystalline variational problems, Bull. Amer. Math. Soc., 84:568-588, 1978.

[TCH92] J.E. Taylor, J.W. Cahn, C.A. Handwerker, Geometric models of crystal growth, Acta Metall., 40: 1443-1474, 1992.

[T84] P. Tolksdorf, Regularity for a more general class of quasilinear elliptic equations, J. Diff. Equ., 51:126-160, 1984.

[WX11] G. Wang, C. Xia, A Characterization of the Wulff Shape by an Overdetermined Anisotropic PDE, Arch. Ration. Mech. Anal., 199:99-115, 2011.

[W01] G. Wulff, Zur Frage der Geschwindigkeit des Wachsthums und der Auflösung der Krystallflachen, Z. Kristallogr. Mineral., 34:449-530, 1901. 